\title{
Saturn's magnetospheric planetary period oscillations, neutral atmosphere circulation, and thunderstorm activity: Implications, or otherwise, for physical links
}

\author{
S. W. H. Cowley ${ }^{1}$ and G. Provan ${ }^{1}$ \\ Received 5 July 2013; revised 3 October 2013; accepted 28 October 2013; published 27 November 2013.
}

[1] Suggestions that the planetary period oscillations (PPOs) observed in Saturn's magnetosphere may be driven or influenced by neutral atmospheric perturbations motivate an exploratory comparison of PPO rotation periods with available tropospheric and stratospheric determinations. Nonpolar atmospheric rotation periods occupy the range $\sim 10.2-10.7 \mathrm{~h}$ associated with the latitudinal jet structure, are similar north and south, and are independent of season, while PPO periods lie in a narrower partly overlapping range $\sim 10.6-10.8 \mathrm{~h}$, are persistently shorter north than south, and undergo a seasonal cycle. In this cycle, widely separated north-south PPO periods during southern summer converge across equinox to values lying within the atmospheric west jet band, remaining well-separated from east jet periods. Closest convergence occurred 1 year post equinox, contemporaneously with the switch in seasonal thunderstorm activity from Southern to Northern Hemispheres. Since most large-scale atmospheric phenomena are related to the west jets, rotating with closely similar periods, they also rotate with periods close to the PPOs under post equinoctial conditions but not otherwise. Specifically, post equinox northern PPOs rotate with a period close to the southern thunderstorms, as well as the north polar spot and hexagon features, while the post equinox southern PPOs rotate with a period close to the pre-equinox northern "string of pearls" and the first colocated post equinox northern thunderstorm, the Great White Spot event. However, even under these conditions, no consistent correspondences in period are found at a detailed level, which taken together with the lack of correspondence at other times does not suggest a direct physical link exists between these phenomena.

Citation: Cowley, S. W. H., and G. Provan (2013), Saturn's magnetospheric planetary period oscillations, neutral atmosphere circulation, and thunderstorm activity: Implications, or otherwise, for physical links, J. Geophys. Res. Space Physics, 118, 7246-7261, doi:10.1002/2013JA019200.

\section{Introduction}

[2] Modulations near the planetary rotation period associated with Saturn's magnetosphere were first detected in Voyager Saturn kilometric radio (SKR) data and have subsequently been studied using remote sensing data from Ulysses and the Hubble Space Telescope (HST) and both remote and in situ data from the Cassini orbiter. These observations have shown that rotating variations occur ubiquitously in SKR, auroral, and plasma wave emissions, as well as in magnetic field and plasma particle data [e.g., Warwick et al., 1981, 1982; Gurnett et al., 1981, 2007, 2010a; Sandel and Broadfoot, 1981; Sandel et al., 1982;

\footnotetext{
${ }^{1}$ Department of Physics and Astronomy, University of Leicester, Leicester, UK.

Corresponding author: S. W. H. Cowley, Department of Physics and Astronomy, University of Leicester, Leicester, LE1 7RH, UK. (swhc1@ion.le.ac.uk)

(C)2013. American Geophysical Union. All Rights Reserved. 2169-9380/13/10.1002/2013JA019200
}

Carbary and Krimigis, 1982; Espinosa and Dougherty, 2000; Cowley et al., 2006; Clarke et al., 2006, 2010a, 2010b; Kurth et al., 2007; Carbary et al., 2007, 2008a, 2008b; Andrews et al., 2008, 2010a, 2011; Nichols et al., 2008, 2010a, 2010b; Burch et al., 2009; Provan et al., 2009a, 2009b; Wang et al., 2010; Ye et al., 2010; Brandt et al., 2010; Badman et al., 2012; Carbary, 2013]. In the absence of a significant tilt of the internal planetary magnetic dipole vector [e.g., Burton et al., 2010], these effects have been ascribed to the occurrence of large-scale systems of electric currents rotating in Saturn's magnetosphere, mapping in the ionosphere to polar auroral latitudes of $\sim 70^{\circ}-75^{\circ}$ [Southwood and Kivelson, 2007, 2009; Andrews et al., 2010a, 2010b; Carbary, 2013]. Two related current systems have been found to be present: one associated with the northern polar region and the other with the southern, which rotate with distinct periods [Kurth et al., 2008; Gurnett et al., 2009a, 2009b; Andrews et al., 2010b; Southwood, 2011; Provan et al., 2011, 2012]. During the initial phase of the Cassini mission from 2004 to 2008, corresponding to Saturn post solstice southern summer conditions, the periods were found to be well-separated: $\sim 10.6 \mathrm{~h}$ for the northern system and $\sim 10.8 \mathrm{~h}$ for the southern, with the southern system 
being stronger than the northern by a factor of $\sim 2.5$ [Provan et al., 2011; Andrews et al., 2012]. It was also found, however, that the periods vary significantly with time [Galopeau and Lecacheux, 2000], converging over a $~ 2$ year interval centered near to Saturn's vernal equinox in mid-August 2009 and reaching near-common values of $\sim 10.66 \mathrm{~h}$ for the northern system and $\sim 10.68$ for the southern in September 2010 [Gurnett et al., 2010b, 2011; Lamy, 2011; Andrews et al., 2012]. The equatorial magnetic oscillation amplitudes of the two systems, and thus by implication the magnitude of the corresponding currents, were also found to reach near-common values in this equinoctial interval [Andrews et al., 2012; Provan et al., 2013]. In early 2011, a new regime of behavior then became established in which the two periods remained close but separate: $\sim 10.64 \mathrm{~h}$ for the northern system and $\sim 10.69 \mathrm{~h}$ for the southern but with small sharp transitions in period and larger transitions in amplitude occurring at 100-200 day intervals, simultaneously in the north and south [Provan et al., 2013]. This behavior continues to the time of writing (mid-2013), almost 3 years after equinox, with five such transitions now being documented. This interval will be referred to below as the post equinox interval of "closely spaced" planetary period oscillation (PPO) periods (2011 to present), as distinct from the pre-equinox interval of more widely separated periods (from prior to 2004 to near the end of 2008), and the interval of convergence between (2009 and 2010).

[3] Although empirical knowledge of these PPO phenomena has thus increased significantly over recent years, their physical origin remains a topic of debate. However, their dual north-south nature discussed above points toward semiindependent polar sources, one in each hemisphere, rather than a single process associated with the magnetospheric equatorial region. One possibility is that they are driven by rotating flow perturbations in the neutral upper atmosphere in the two polar regions [Smith, 2006, 2010], specifically in the Pedersen-conducting region of the thermosphere at altitudes $\sim 1000 \mathrm{~km}$ above the 1 bar reference level [Galand et al., 2011]. Such atmospheric flow perturbations will then drive a rotating flow and current system in the ionosphere through ion-neutral collisions, in turn communicated via field-aligned currents to the magnetosphere [Jia et al., 2012; Jia and Kivelson, 2012]. The required atmospheric flow perturbations vary with azimuth $\varphi$ and time $t$ as $e^{j(\Phi(t)-m \varphi)}$ with $m=1$, corresponding to a twin-vortex flow pattern in each polar region that rotates with time at the PPO period $\tau=2 \pi /(d \Phi / d t)$.

[4] It is evident, however, that the rotation periods concerned are not directly those of the neutral thermosphere itself. The plasma is known to significantly subcorotate on outer auroral field lines from observations in both the magnetosphere [Thomsen et al., 2010; Arridge et al., 2011] and the ionosphere [Stallard et al., 2004, 2012], and this will inescapably lead to lesser but still significant subcorotation in the relevant region of the thermosphere. Numerical modeling studies of the effects of ion-neutral drag suggest that the subcorotation of Saturn's thermosphere will be approximately half that of the plasma [Smith and Aylward, 2008; Galand et al., 2011]. Since the angular velocity of the plasma on auroral field lines is typically $\sim 50 \%$ of that of rigid corotation, or less, as indicated by the above cited studies, the angular velocity of the thermosphere will typically be $\sim 75 \%$ of rigid corotation or less. Taking rigid corotation to correspond to a period of $\sim 10.6 \mathrm{~h}$, as discussed further in section 2 , the rotation period of the plasma is thus $\sim 21 \mathrm{~h}$ or greater, while that of the auroral thermosphere is $\sim 14 \mathrm{~h}$ or greater. The latter value is thus well removed from that of the PPO periods as indicated above. In addition, the magnetospheric and hence thermospheric flows in these regions are expected to respond strongly to magnetospheric dynamics such as the effects of major compressions imposed by the solar wind [e.g., Cowley et al., 2005], thus leading to considerable variability in the thermospheric rotation period. On the contrary, however, the PPO periods show no major variability on solar wind time scales of tens of days [e.g., Badman et al., 2008; Gurnett et al., 2011; Lamy, 2011; Andrews et al., 2012; Provan et al., 2013], though $\sim 1 \%$ fluctuations associated with changes in solar wind speed have been reported by Zarka et al. [2007].

[5] A further possibility, however, is that the perturbations could be generated by asymmetric phenomena in the lower atmosphere with " $m=1$ " harmonic content [Smith, 2006], whose effects are coupled to the thermosphere by upward viscous or wave momentum transport from the mesosphere, stratosphere, or even troposphere [e.g., Matcheva and Barrow, 2012]. Indeed, Fischer et al. [2012, 2013] have suggested a close connection between variations in the PPO periods and the occurrence of the 2010-2011 Great White Spot (GWS) tropospheric thunderstorm event. In this case the relevant period is expected to be that of the lower neutral atmosphere and its embedded dynamical systems. In response to such suggestions, in this paper we present an exploratory empirical discussion of the relation between the PPO rotation periods and those of the neutral atmosphere (section 2) and its dynamical phenomena (sections 3 and 5), paying particular attention to PPO effects around the time of the 2010-2011 GWS storm (section 4).

\section{PPO Periods and the Rotation Period of Saturn's Neutral Atmosphere}

\subsection{Atmospheric Rotation Periods at Cloud Height and Above}

[6] Measurements of the rotation period of Saturn's atmosphere have been obtained from the motions of cloud features observed in planetary images in the visible and infrared (IR) wavebands (see, e.g., the review by Del Genio et al. [2009, and references therein]). The first such data were derived from Voyager images obtained during the flybys in 1980-1981 [e.g., Ingersoll et al., 1984; Sánchez-Lavega et al., 2000], followed by HST observations in 1990-2004 [e.g., Barnet et al., 1992; Sánchez-Lavega et al., 2004] and Cassini Imaging Science Subsystem (ISS) and Visual and Infrared Mapping Spectrometer (VIMS) observations since 2004 [e.g., Vasavada et al., 2006; Choi et al., 2009]. Features observed in scattered sunlight in visible and near-IR images generally correspond to ammonia-ice clouds and haze in the upper troposphere, at pressure levels from $\sim 100$ mbar down to $\sim 1$ bar, below which sunlight does not penetrate $(1 \mathrm{bar}=100 \mathrm{kPa})$. Observations in the mid-IR (e.g., VIMS at $\sim 5 \mu \mathrm{m}$ ) then reveal deeper ammonium hydrosulfide clouds at pressures of $\sim 2.5-4.5$ bars, silhouetted against planetary thermal emissions. The hypothesized water ice and aqueous water-ammonia clouds 


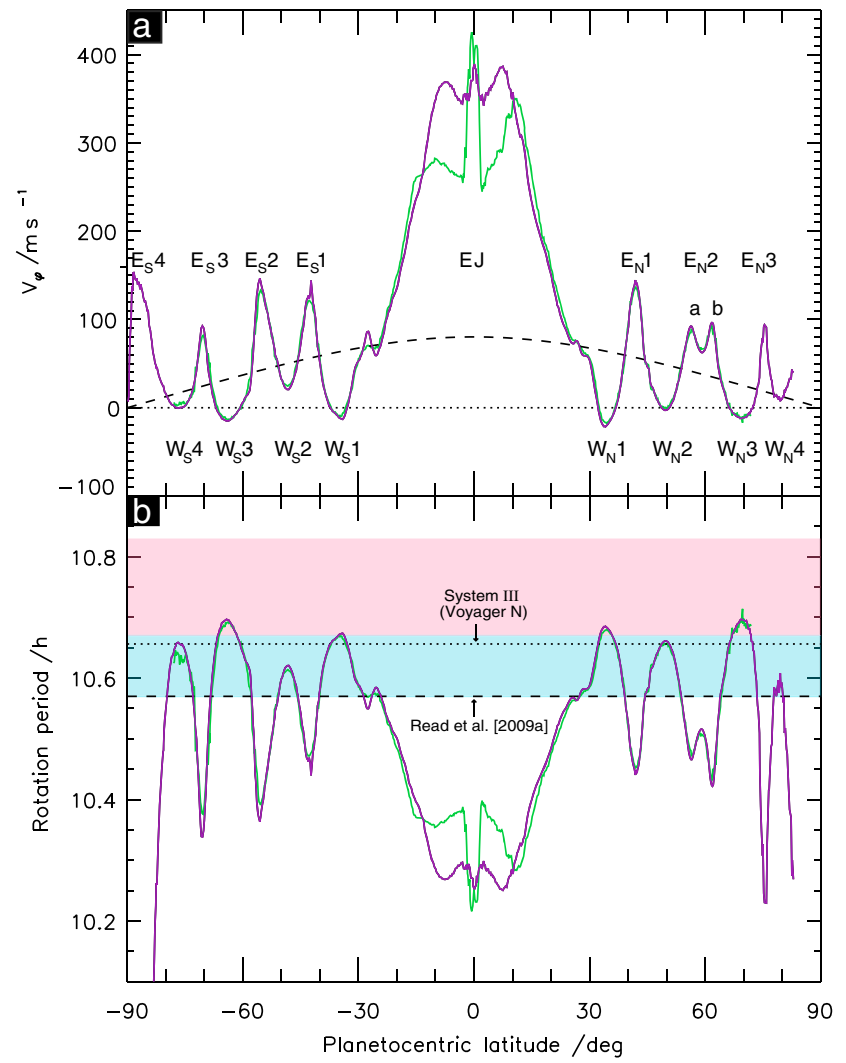

Figure 1. Plots showing (a) upper tropospheric zonal (azimuthal) wind speeds relative to System III taken from García-Melendo et al. [2011] and (b) corresponding atmospheric rotation periods, both plotted versus planetocentric latitude. The wind speeds were determined from the motions of cloud/haze structures observed in pairs of Cassini/ISS images obtained during 2004-2009 using near-IR continuum (purple lines) and methane absorption band (green lines) filters (see Garcia-Melendo et al. [2011] for details). The purple lines correspond to atmospheric pressures $\sim 350-700$ mbar, while the green lines correspond to a pressure of $\sim 60$ mbar near the equator, and $\sim 250$ mbar at higher latitudes. $\mathrm{E}_{\mathrm{N}, \mathrm{S}} 1, \mathrm{E}_{\mathrm{N}, \mathrm{S}} 2$, and so on, mark the east (prograde) jet peaks in the Northern $(\mathrm{N})$ and Southern (S) Hemispheres, while $\mathrm{W}_{\mathrm{N}, \mathrm{S}} 1, \mathrm{~W}_{\mathrm{N}, \mathrm{S}} 2$, and so on, similarly mark the west (retrograde) jet peaks. EJ indicates the equatorial eastward jet. The dotted line in Figure $1 \mathrm{~b}$ shows the System III period based on the Voyager north SKR modulation period, which represents the dotted zero baseline in Figure 1a. The dashed lines in both panels correspond to the deep planetary rotation period estimated by Read et al. [2009a]. The blue and pink bands in Figure $1 \mathrm{~b}$ show the range of northern and southern PPO periods, respectively, observed during the Cassini mission to date.

at $\sim 10-20$ bars, which are the presumed source of the thunderstorm and GWS activity to be discussed in sections 3-5, are not accessible to direct observation (see, e.g., the review by West et al. [2009, and references therein]).

[7] It should thus be noted that the cloud layers that provide detailed data on atmospheric rotation are far removed in altitude from that of the main Pedersen-conducting layer in the ionosphere, located in the thermosphere $\sim 1000 \mathrm{~km}$ above the 1 bar level, corresponding to pressures of $\sim 10$ nbar
[Galand et al., 2011]. Specifically, the water condensation cloud layer lies $\sim 200 \mathrm{~km}$ below the 1 bar level, the ammonium hydrosulfide clouds $\sim 50 \mathrm{~km}$ below, and typical ammonia-ice cloud tops $\sim 50 \mathrm{~km}$ above, compared with the tropopause ( $\sim 80$ mbar) at $\sim 100 \mathrm{~km}$, the stratopause $(\sim 0.1 \mathrm{mbar})$ at $\sim 500 \mathrm{~km}$, and the mesopause $(\sim 100 \mathrm{nbar})$ at $\sim 800 \mathrm{~km}$, giving way to the thermosphere above. (We note that the International Astronomical Union (IAU) Saturn 1 bar reference spheroid has equatorial and polar radii of 60,268 and $54,364 \mathrm{~km}$, respectively, and that in the lower atmosphere where the temperature is $\sim 100-150 \mathrm{~K}$, the atmospheric scale height is $\sim 50 \mathrm{~km}$.) Nevertheless, despite the spatial separation, it is evident that the PPO periods must be driven in some manner by the deep rotation of the planet, coupled through these lower atmospheric layers, such that comparison of atmospheric and PPO rotation periods is of relevance.

[8] Figure 1a shows zonal (azimuthal) wind velocities determined by García-Melendo et al. [2011] from crosscorrelation analysis of longitudinal brightness scans of pairs of Cassini ISS images obtained during 2004-2009, plotted versus planetocentric latitude (employed, as opposed to planetographic latitude, throughout this paper). Results are shown for images obtained using two near-IR filters that are sensitive to clouds at somewhat different upper tropospheric heights, at pressures $\sim 350-700$ mbar for the purple curve and $\sim 250$ mbar for the green curve, the latter increasing to $\sim 60$ mbar in the equatorial region where clouds and haze extend to higher altitudes. The data cover nearly the full range of planetary latitudes, except for the immediate vicinity of the North Pole, where Cassini VIMS observations have revealed the existence of a very similar cyclonic wind structure to that shown in the south [Baines et al., 2009]. The wind velocities are shown relative to the IAU System III rotating frame and assume that the observations refer (to sufficient approximation) to the 1 bar reference spheroid. The System III frame rotates with a period of exactly $10 \mathrm{~h} 39 \mathrm{~m} 22.4 \mathrm{~s}(10.6562 \mathrm{~h})$. As discussed in section 2.2 below, this corresponds to the modulation period of northern SKR emissions observed during the Voyager Saturn encounters, assumed at that time to represent the deep rotation period of the planet. Despite the more recent results outlined in section 1 which show this is not the case, this frame continues to be employed as a convenient standard in atmospheric studies. We note that the Voyager 1 SKR period published by Desch and Kaiser [1981] of $10 \mathrm{~h} 39 \mathrm{~m} 24 \pm 7 \mathrm{~s}$ $(10.6567 \pm 0.0019 \mathrm{~h})$ was amended to the above slightly different System III value and communicated to the IAU in 1982 [Davies et al., 1983]. The values of these and other significant rotation periods are collected together for easy reference and comparison in Table 1.

[9] Figure 1a shows that Saturn's zonal upper tropospheric winds consist of an eastward (prograde) equatorial "jet" ("EJ" in the figure) spanning $\sim \pm 20^{\circ}$ latitude about the equator, flanked at higher latitudes by a system of eastward and westward jets labeled $\mathrm{E}_{\mathrm{N}} 1, \mathrm{~W}_{\mathrm{N}} 1$, and so on in the north, and $E_{S} 1, W_{S} 1$, and so on in the south, as indicated in the figure. The wind speed in the equatorial jet peaks at $\sim 300-400 \mathrm{~m} \mathrm{~s}^{-1}$ in the System III frame, with differences between the purple and green curves indicating the presence of vertical wind shear. The east jets have lesser peak wind speeds $\sim 100-150 \mathrm{~m} \mathrm{~s}^{-1}$ in this frame, while the west jets have near-zero peak speed, neither showing evidence of significant variation with height 
COWLEY AND PROVAN: MAGNETOSPHERIC OSCILLATIONS AND THE GWS

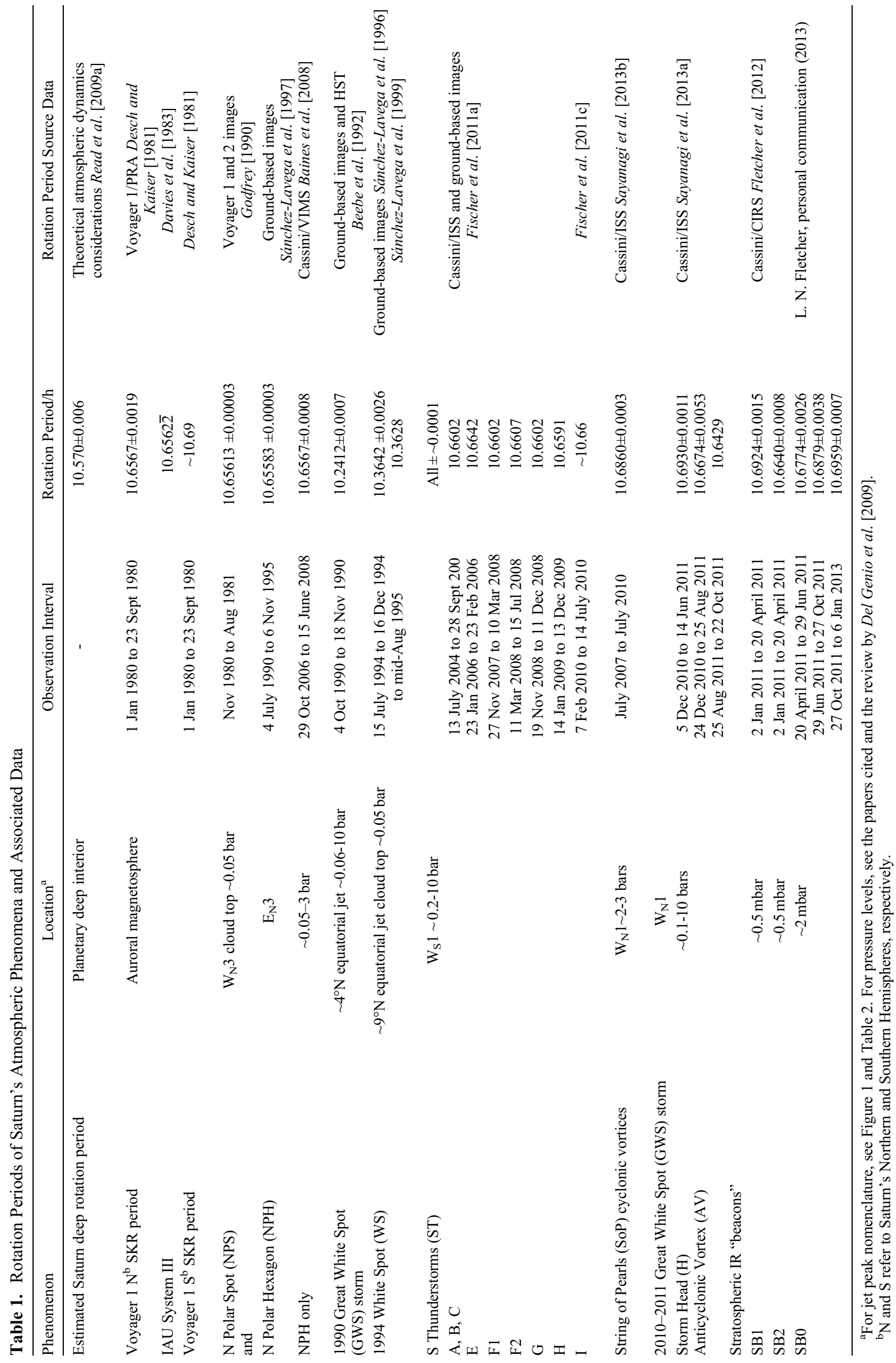


Table 2. Planetocentric Latitudes and Atmospheric Rotation Periods at Tropospheric Jet Peaks ${ }^{\mathrm{a}}$

\begin{tabular}{|c|c|c|c|c|c|}
\hline North Peaks ${ }^{\mathrm{b}}$ & Latitude/deg & Period/h & South Peaks ${ }^{\mathrm{b}}$ & Latitude/deg & Period/h \\
\hline Equatorial jet center & 0 & 10.252 & - & - & - \\
\hline Equatorial jet north peak & 7 & 10.250 & Equatorial jet south peak & -7 & 10.268 \\
\hline $\mathrm{W}_{\mathrm{N}} 1$ & 34 & 10.686 & $\mathrm{~W}_{\mathrm{S}} 1$ & -34 & 10.674 \\
\hline $\mathrm{E}_{\mathrm{N}} 1$ & 42 & 10.441 & $E_{S} 1$ & -42 & 10.440 \\
\hline $\mathrm{W}_{\mathrm{N}} 2$ & 50 & 10.661 & $\mathrm{~W}_{\mathrm{S}} 2$ & -48 & 10.621 \\
\hline $\mathrm{E}_{\mathrm{N}} 2 \mathrm{a}$ & 56 & 10.466 & $\mathrm{E}_{\mathrm{S}} 2$ & -56 & 10.364 \\
\hline$E_{N} 2 b$ & 62 & 10.423 & & & \\
\hline $\mathrm{W}_{\mathrm{N}} 3$ & 69 & 10.697 & $\mathrm{~W}_{\mathrm{s}} 3$ & -64 & 10.697 \\
\hline $\mathrm{E}_{\mathrm{N}} 3$ & 75 & 10.230 & $\mathrm{E}_{\mathrm{S}} 3$ & -71 & 10.338 \\
\hline $\mathrm{W}_{\mathrm{N}} 4$ & 80 & 10.608 & $\mathrm{~W}_{\mathrm{S}} 4$ & -77 & 10.659 \\
\hline $\mathrm{E}_{\mathrm{N}} 4$ & $88^{\mathrm{c}}$ & $\sim 7.2^{\mathrm{c}}$ & $\mathrm{E}_{\mathrm{S}} 4$ & -88 & 6.746 \\
\hline
\end{tabular}

${ }^{\mathrm{a}}$ Determined from Cassini/ISS cloud data presented by Garcia-Melendo et al. [2011].

${ }^{\mathrm{b}}$ For jet peak nomenclature, see Figure 1.

${ }^{c}$ Estimated from Figure 12 of Baines et al. [2009].

over the more restricted altitude range in these cases. The nearzero speed of the west jets indicates that their rotation period is close to that of the Voyager north SKR period, as will be discussed further in section 2.2.

[10] In Figure 1b, these zonal velocities have been transformed to atmospheric rotation periods, again plotted versus latitude. The rotation periods in the central part of the equatorial jet $\left(\sim \pm 10^{\circ}\right.$ latitude) are typically $\sim 10.25$ $10.30 \mathrm{~h}$ (purple curve), while the periods of the peaks of the east jets lie in the range $\sim 10.35-10.45 \mathrm{~h}$, with the exceptions of $\mathrm{E}_{\mathrm{N}} 3$ also with a period of $\sim 10.25 \mathrm{~h}$ and the near-polar peaks $\mathrm{E}_{\mathrm{N}, \mathrm{S}} 4$ that have a much smaller period of $\sim 7 \mathrm{~h}$ (off scale). The periods of the peaks of the west jets, by comparison, lie in the range $\sim 10.60-10.70 \mathrm{~h}$. The individual atmospheric rotation periods at the east and west jet peaks, specifically for the purple curves in Figure 1, are recorded for future reference in Table 2 . For typical $\sim 5 \mathrm{~m} \mathrm{~s}^{-1}$ uncertainties in the zonal speeds noted by Garcia-Melendo et al. [2011], the estimated uncertainties in period are $\sim \pm 0.005 \mathrm{~h}$, such that the values are recorded in hours to three decimal places. The dotted line in the figure shows the System III period given above, which lies close to the west jet peaks as expected from Figure 1a. The dashed line shows an estimate of Saturn's deep rotation period determined by Read et al. [2009a] from atmospheric dynamics considerations. This period is $10 \mathrm{~h} 34 \mathrm{~m} 13 \pm 20 \mathrm{~s}(10.570 \pm 0.006 \mathrm{~h})$, lying more centrally between the periods of the east and west jets. The dashed line in Figure 1a shows the corresponding zonal velocity of this frame relative to System III.

[11] Given the seasonal variability of the PPO periods outlined in section 1 (discussed in more detail in section 2.2), it is of interest to consider the related variability of these atmospheric winds. We first note that the Cassini ISS data employed to construct Figure 1a span 2004-2009, corresponding to post solstice southern summer to near vernal equinox. Examination of subsets of these data, however, reveals no significant wind variations over this interval [García-Melendo et al., 2011]. Similar results have been obtained from comparison with both Voyager and HST data, thus indicating overall stability of the wind pattern over 30 year time scales, independent of Saturn's seasons (e.g., García-Melendo et al. [2011] and section 6.3.5 of Del Genio et al. [2009]). This lack of seasonality is not completely surprising, however, since the most significant energy input to these layers comes steadily from the interior of the planet rather than seasonally from the Sun. The only exception to these statements concerns the equatorial jet, which has appeared to show significant reductions in flow speed from peak Voyager values of $\sim 450 \mathrm{~m} \mathrm{~s}^{-1}$ [e.g., Sánchez-Lavega et al., 2000], down to $\sim 275 \mathrm{~m} \mathrm{~s}^{-1}$ in ground-based and HST data obtained for several years following the 1990 GWS [e.g., Sánchez-Lavega et al. 2004] (see section 3.4). The implied change in equatorial rotation period is from $\sim 10.19$ to $\sim 10.37 \mathrm{~h}$. It remains unclear, however, how much of this reduction was due to real temporal change and how much to the presence of higher clouds and hazes in the postGWS interval, given the significant vertical wind shear in this region indicated in Figure 1 [Sayanagi and Showman, 2007].

[12] The pink and blue bands in Figure $1 \mathrm{~b}$ then show the ranges of the PPO periods for the north and south system oscillations, respectively, as observed during the Cassini mission to date (shown versus time in Figure $2 \mathrm{~b}$, introduced in section 2.2). The top of the pink band at $10.83 \mathrm{~h}$ and the bottom of the blue band at $10.57 \mathrm{~h}$ correspond to the condition of wide separation of the PPO periods observed by Cassini in 2008, prior to vernal equinox, while the period where these bands meet at $\sim 10.67 \mathrm{~h}$ corresponds to the condition of near-coalesced PPO periods observed post equinox in late 2010. We note that the Voyager SKR observations correspond to post vernal equinox conditions one Saturn year earlier (see Figure 2b), such that, in agreement with the Cassini results, the System III period (dotted line) based on the Voyager north SKR period also lies near the top of the blue band. Overall, it can be seen that the north PPO period spans a range from between the periods of the east and west jet peaks corresponding closely to the deep rotation period estimated by Read et al. [2009a] to the peaks of the west jets. The south PPO period spans a slightly larger range starting at the latter value and extending to periods in excess of any associated with the neutral atmosphere at cloud level.

\subsection{PPO Rotation Periods and Their Seasonal Variation}

[13] In Figure 2, we now focus on the PPO periods and their seasonal variation, showing their values intermittently determined over a $\sim 35$ year interval spanning more than a full Saturn year, together with the rotation periods of some atmospheric features as discussed below. Figure 2 a first plots the latitude of the Sun at Saturn showing the seasons, with equinoxes and solstices marked by the vertical dotted lines. 
Equinox Solstice Equinox Solstice Equinox

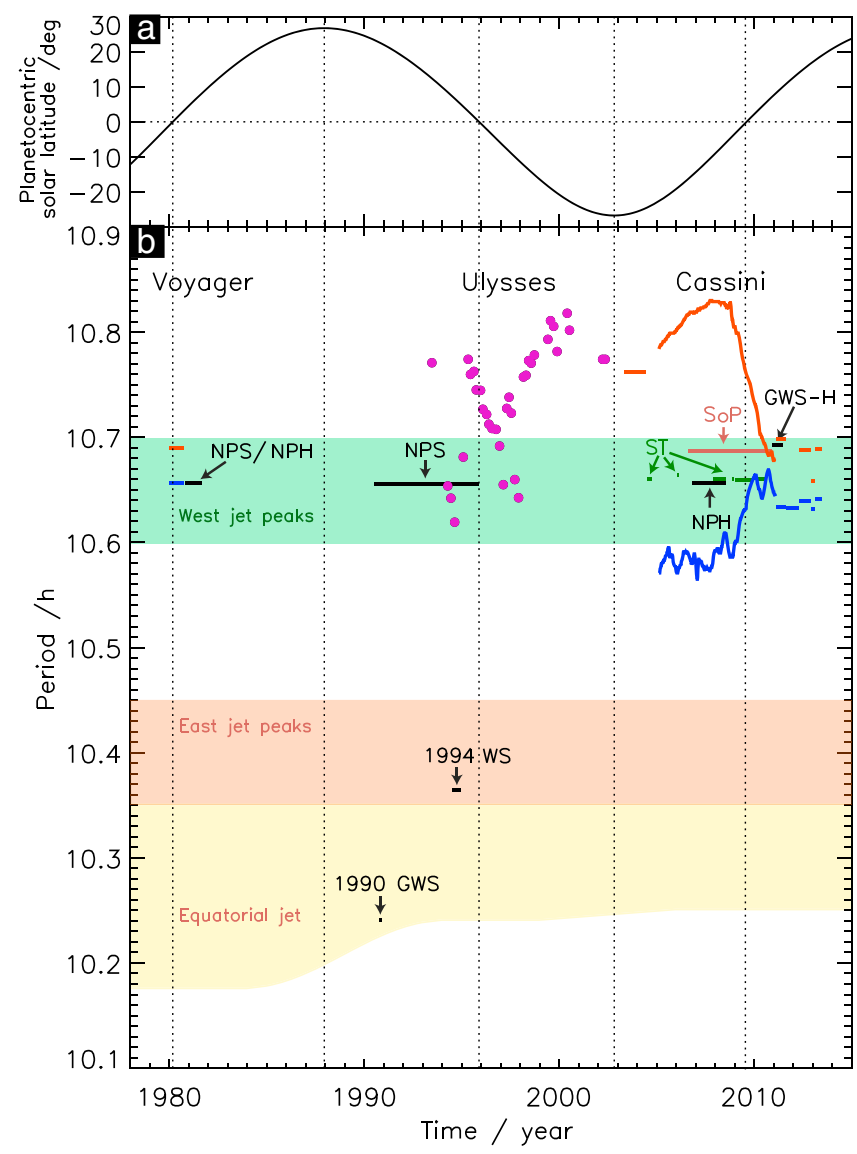

Figure 2. Plots showing (a) the planetocentric latitude of the Sun at Saturn and (b) the PPO rotation periods derived from Voyager, Ulysses, and Cassini measurements as indicated, together with the rotation periods of various atmospheric features, over a $\sim 35$ year interval spanning more than a full Saturn year. The vertical dotted lines indicate Saturn's seasons, specifically the northern and southern summer solstices where the solar latitude is maximum and minimum, respectively, and the equinoxes where the latitude passes through zero. The northern and southern PPO periods determined from Voyager and Cassini data are shown by the blue and red lines, respectively, while the Ulysses data are shown by purple dots since the corresponding hemisphere remains undetermined from direct measurement of their polarization. The green and orange horizontal bands show the range of atmospheric rotation periods corresponding to the west and east jet peaks in the upper troposphere, respectively, while the yellow band shows the extension of the latter into the central equatorial eastward jet (see Figure 1). The black, green, and orange lines show the rotation periods of various atmospheric phenomena over their intervals of observation. Specifically, we show the periods and observation intervals for the north polar spot (NPS) and north polar hexagon (NPH) (black lines), the 1990 Great White Spot storm (1990 GWS) and 1994 White Spot (1994 WS) (black lines), the western edge of the head of the 2010-2011 Great White Spot storm (GWS-H) (black line), the Cassini era southern thunderstorms (ST) (green lines), and the Cassini era "string of pearls" (SoP) phenomenon (orange line).
Vernal equinox in the Voyager era occurred on 3 March 1980, northern summer solstice on 11 December 1987, autumnal equinox during the Ulysses measurements on 19 November 1995, southern summer solstice in the preCassini era on 27 October 2002, and vernal equinox during the Cassini era on 11 August 2009. The PPO periods are shown in the upper part of Figure $2 b$, determined from Voyager, Ulysses, and Cassini measurements as indicated. The Voyager periods are those of Desch and Kaiser [1981] (Table 1), obtained from Voyager 1 SKR data during approach to the planet from January to September 1980 spanning vernal equinox (closest approach being mid-November 1980). The principal period shown by the blue bar spanning this interval corresponds to the north PPO period associated with dominant right-hand polarized emissions, which with slight modification noted above forms the basis of IAU System III. A second statistically significant period at $10.69 \mathrm{~h}$ obtained from the same SKR data is taken to correspond to the southern PPO period, shown by the red bar, indicating closely spaced periods similar to those observed post equinox in Cassini data. The periods determined from distantly sensed Ulysses SKR observations presented by Gurnett et al. [2010b] [see also Galopeau and Lecacheux, 2000] are shown by the purple dots in the figure, since the corresponding hemisphere indicated by emission polarization remains undetermined. However, from the context of the Cassini data, it seems reasonable to infer that the longer periods correspond to the southern system and the shorter to the northern, at least for the data post autumnal equinox. The Cassini data first consist of a south PPO period of $10.7625 \pm 0.0100 \mathrm{~h}$ determined by Gurnett et al. [2005] from SKR data obtained during approach to the planet between April 2003 and June 2004, shown by the red bar spanning this post solstice interval. From near the beginning of 2005 , the blue and red lines then show the north and south PPO periods determined from in-orbit Cassini magnetic field data by Andrews et al. [2012] and Provan et al. [2013], here brought up-to-date to mid-2013.

[14] As outlined in section 1, the Ulysses and Cassini data indicate well-separated PPO periods during southern summer, which converged across equinox, and then remained relatively closely spaced in the recent northern spring interval (though notably then at variance with the behavior in the equivalent southern spring interval covered by Ulysses). Indeed, these data indicate a double-peaked profile for the PPO south period over the southern summer season, with a relative minimum around solstice, while the variation of the north period over this interval remains rather less well determined. In addition, it is seen that the near-equinoctial Voyager periods are closely similar to those observed one Saturn year later during the Cassini era.

[15] The correspondence between Voyager and Cassini PPO periods is shown in greater detail in Figure 3, where we show a zoomed-in plot in a similar format to Figure 2, beginning just before Cassini era vernal equinox (and also spanning the 2010-2011 GWS storm discussed in section 4). The Cassini PPO periods are again shown by the blue and red lines in the figure, illustrating the change in character of the PPO phase data that took place near the beginning of 2011. Prior to this time, the orbit-to-orbit phase values determined from the magnetic field data evolved smoothly with time and were analyzed by Andrews et al. [2012] using running 


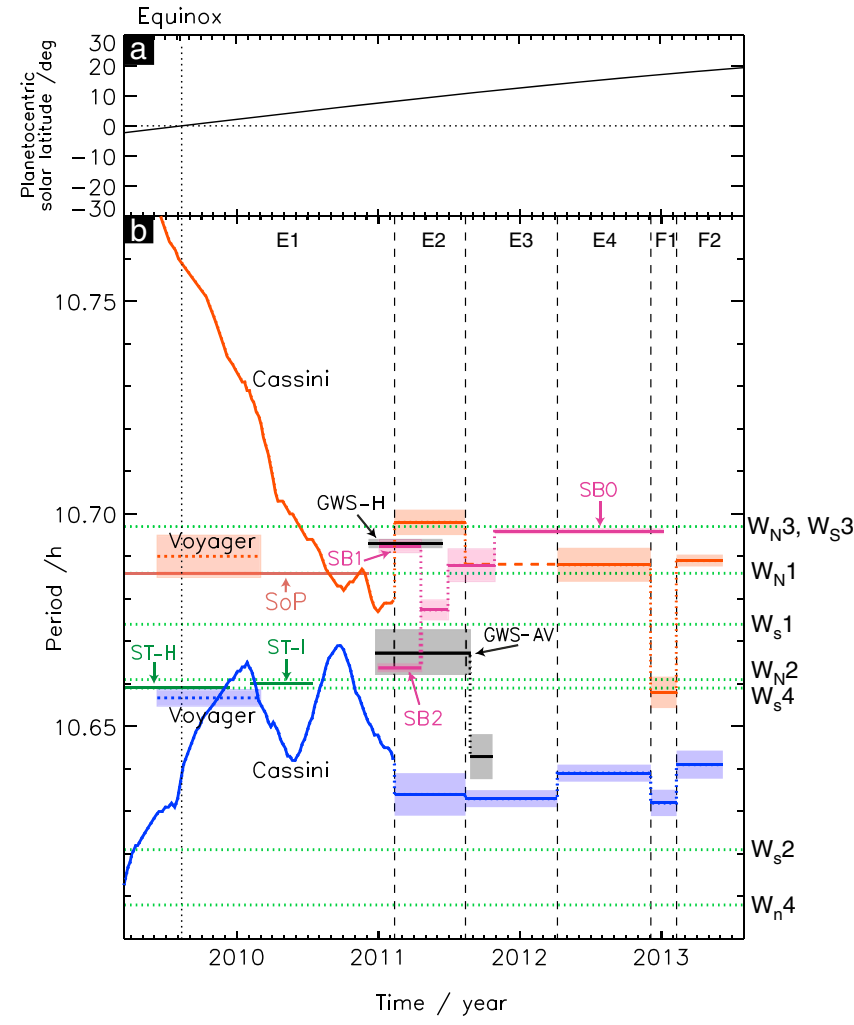

Figure 3. Zoomed-in view of a portion of Figure 2, showing (a) the planetocentric latitude of the Sun at Saturn and (b) the rotation periods of the PPOs determined from Cassini magnetic field data together with various atmospheric features, over a $\sim 4$ year interval from mid-2010 to mid-2013 spanning vernal equinox (vertical dotted line) and northern spring. The format is similar to Figure 2, except that the rotation periods corresponding to individual west jet peaks are shown by the horizontal green dotted lines, as marked to the right of the figure. The horizontal red and blue dotted lines in Figure $3 \mathrm{~b}$ also show the north and south Voyager SKR periods transposed through one Saturn year, together with shaded uncertainty intervals. Additional horizontal lines and shading show the periods and intervals of various atmospheric features, specifically for southern thunderstorms $\mathrm{H}$ and I (ST-H and ST-I) (green solid lines), the northern "string of pearls" feature (SoP) (orange line), the western edge of the head of the 2010-2011 Great White Spot storm (GWS-H) (black line), the associated anticyclonic vortex or "dark spot" (GWS-AV) (black lines), and the stratospheric anticyclonic "beacons" (SB1, SB2, and SB0) (purple lines).

25-point data sets typically spanning $\sim 200$ days (similar to corresponding SKR determinations). After this time, however, the magnetic data show abrupt changes in amplitude and phase behavior every $\sim 100-200$ days, each such interval being analyzed using piecewise linear fits to the phases over the intervals between the changes shown by the vertical dashed lines in the figure [Provan et al., 2013]. This procedure results in piecewise intervals of constant period as shown, together with uncertainty intervals indicated by the shading. The Voyager periods in Figure $2 b$ have then been transposed through one Saturn year and are shown by the blue and red dotted lines and shading. The dominant north
Voyager period (blue) is clearly in very close agreement with the Cassini values over the corresponding interval. The second Voyager period, assumed to be the south period (red), is also in approximate agreement with the rapidly falling Cassini values, particularly later in the (transposed) interval when Voyager 1 was nearer to the planet during the approach interval.

[16] Figures $2 \mathrm{~b}$ and $3 \mathrm{~b}$ also indicate the rotation periods of the atmospheric jets for purposes of comparison. In Figure $2 b$, the green band corresponds to the periods of the west jet peaks, the orange band to the period of the east jet peaks, both unvarying with time over the interval according to presently available information, while the yellow band indicates the extension of the east jet peak periods into those of the equatorial jet, which may possibly be somewhat variable with time as mentioned above. In Figure 3b, the horizontal green dotted lines similarly show the rotation periods of individual west jet peaks as marked to the right of the diagram (Table 2), where we recall the approximate $\pm 0.005 \mathrm{~h}$ uncertainties in these values. The east and equatorial jet periods are, of course, well off scale in Figure 3b, toward lower values.

[17] These results further demonstrate the relation of the PPO periods with the rotation periods of the west jet peaks. The well-separated PPO periods that occur under southern summer conditions lie outside the range of periods of the west jet peaks at shorter periods for the northern system, while remaining significantly longer than those of the east jet peaks and at longer periods for the southern system. Their mean value, however, near $10.7 \mathrm{~h}$, lies close to the period of the west jet peaks, albeit at the top of the band. The two PPO periods then converge across equinox into the band of periods corresponding to the west jets, the northern period into the central and lower part of the band, and the southern period into the upper part of the band. It is for this reason and the accidental fact that the Voyager Saturn encounter occurred under such equinoctial conditions that the dominant northern period used to define System III happened to lie close to the rotation period of the west jets. We note, however, that although these empirical correspondences in period are clear, the implications for possible physical connections, if any at a detailed level, remain unclear.

[18] It is nevertheless evident from the fact that the southern PPO oscillations extend in period to values well beyond any associated with the neutral atmosphere at cloud level that they cannot be driven in a straightforward way by rotating perturbations communicated upward (e.g., by waves) from lower atmospheric levels into the thermosphere, mentioned as one possibility by Smith [2006]. This follows from the fact that lower atmospheric disturbances with potential $m=1$ harmonic content generally rotate closely with the zonal flow at their corresponding latitude (see section 3 ), and there is no layer, at cloud level at least, that rotates with the period of the longer-period southern PPOs during southern summer. More generally, the above discussion provides no evidence of possible agency in the lower atmosphere that could account for either the observed north-south asymmetry in the PPO rotation periods or for their significant seasonal dependency, given the approximate north-south symmetry and observed strong seasonal stability of the atmospheric flow. These properties of the PPOs must thus arise from other agencies, such as the hemispheric planetary magnetic field asymmetry, and the seasonal conductivity of the ionosphere. 
[19] Of course, the atmospheric wind speeds discussed in this section pertain directly only to tropospheric levels up to $\sim 100$ mbar, corresponding to $\sim 100 \mathrm{~km}$ above the 1 bar level. However, the wind profile can be extended upward into the stratosphere using temperature data and theoretical considerations. Results using Cassini Composite Infrared Spectrometer (CIRS) data suggest that the jet structure in Figure 1 becomes slowly attenuated with altitude but remains intact in form down to pressure levels of at least $\sim 1$ mbar $(\sim 300 \mathrm{~km})$ and possibly beyond [Fouchet et al., 2008; Read et al., 2009b]. However, no flow information, direct or inferred, is available at middle atmospheric levels above $\sim 500 \mathrm{~km}$.

\section{Rotation Periods of Atmospheric Phenomena in Relation to PPOs}

[20] In this section we now discuss the observed relationships between PPO periods and those of various atmospheric phenomena, preparatory to discussing relationships with the 2010-2011 GWS in section 4. As we will see, most of such large-scale phenomena are related specifically to the west jets, which given the findings in section 2 , leads directly to close correspondences between the periods of atmospheric phenomena and those of PPOs under post equinox conditions.

\subsection{The North Polar Spot and Hexagon}

[21] A prominent atmospheric feature observed at northern polar latitudes, discovered in Voyager image data and the first to be discussed in relation to PPO phenomena, specifically the equinoctial Voyager north SKR modulation, is the north polar hexagon (NPH) cloud feature and its sometimeassociated north polar spot (NPS) [Godfrey, 1988]. The $\mathrm{NPH}$ encircles the northern pole at $\sim 75^{\circ}-77^{\circ}$ latitude, thus lying within northern east jet $\mathrm{E}_{\mathrm{N}} 3$, where the zonal wind speeds are $\sim 100 \mathrm{~m} \mathrm{~s}^{-1}$ relative to System III (see Figure 1 and Table 2). The NPS, the largest anticyclonic oval cloud feature yet observed at Saturn, $\sim 7000 \mathrm{~km}$ east-west by $\sim 5000 \mathrm{~km}$ north-south, then lay equatorward of one of the faces of the hexagon, centered at $\sim 71^{\circ}-72^{\circ}$ latitude. (We note that $1000 \mathrm{~km}$ north-south corresponds to $\sim 1^{\circ}$ of latitude at Saturn.) The NPS was thus centered in the northern part of west jet $\mathrm{W}_{\mathrm{N}} 3$, near where the mean zonal velocity passes through zero relative to System III on the poleward side of the west jet peak (Figure 1a). Both the NPS and the NPH structure were then found to be near-stationary in the System III frame, with a relative eastward velocity of only $\sim 0.1 \mathrm{~m} \mathrm{~s}^{-1}$, while the winds within the NPH were found to flow around the hexagonal streamlines at $\sim 100 \mathrm{~m} \mathrm{~s}^{-1}$ [Godfrey, 1990], corresponding to the east jet structure. The very low relative velocity means that the rotation periods of the NPS and NPH were very close indeed to that of the north PPOs during the Voyager encounter, being determined by Godfrey [1990] to be $10.65613 \pm 0.00003 \mathrm{~h}$ (Table 1) as indicated by the black bar labeled NPS/NPH in Figure 2b, thus lying well within the error bars of the Desch and Kaiser [1981] SKR value. This suggested a close physical connection between these phenomena, thought at the time to be Saturn's deep rotation period.

[22] Ground-based observations of the NPS between early July 1990 and early November 1995 indicated a slightly shorter period during this interval of $10.65583 \pm 0.00003 \mathrm{~h}$, though still lying well within the errors of the Voyager north
PPO period, and hence very close to the System III period [Sánchez-Lavega et al., 1997]. This is shown by the central black bar labeled NPS in Figure $2 b$ during the early Ulysses interval, where we see that the value is not inconsistent with a few contemporaneous scattered low-period PPO values determined from Ulysses data. These observations also confirmed the long-lived nature of both the NPH and NPS features, over $\sim 15$ years during the northern summer season.

[23] During the following southern summer season, however, northern polar latitudes became inaccessible to ground observations and permanently in shadow but were observed on high-latitude Cassini orbits during 2006-2008 in planetary thermal IR emission. Observations by both Cassini VIMS and CIRS confirmed the continued existence of the hexagon within upper tropospheric layers (not extending into the stratosphere), thus continuing in time essentially over a whole Saturn year independent of season [Fletcher et al., 2008; Baines et al., 2009]. Amateur astronomer images continue to show its presence at the time of writing (mid-2013) (D. P. Milika and P. Nicholas, personal communication, 2013). However, no evidence has been found for the continued presence of the NPS. Measurements of the orientation of the hexagon in VIMS data obtained from late October 2006 to mid-June 2008, however, indicated a rotation period which was almost unchanged compared with earlier values, at least to within $\sim 0.001 \mathrm{~h}$ [Baines et al., 2008]. This is shown by the black bar marked NPH on the right side of Figure $2 b$, from which it is clear that no correspondence then existed with the contemporaneous southern summer north system PPO period. This finding shows that there is in fact no direct physical connection between the NPS/NPH and the north PPO oscillations, since the former rotates with a period almost invariant over the Saturn year, like the mean winds of the jets themselves, while the latter undergoes a seasonal variation, only approaching the NPS/NPH period post equinox. The close correspondence during the Voyager flybys was thus an accidental consequence of the post equinox conditions than prevailing.

\subsection{Thunderstorm Activity}

[24] A more intermittent phenomenon observed in Saturn's atmosphere is the occurrence of thunderstorms, having spatial scales $\sim 1000-3000 \mathrm{~km}$ (e.g., section 6.4 .2 of the review by Del Genio et al. [2009]). These erupt abruptly on time scales of a day or less, last for variable intervals of a few tens of days, and may occur either singly or in groups spanning many months, possibly with intervals of many months between (see section 5 below). At near-IR wavelengths, they are observed initially as bright structured clouds, presumed to be formed by rapid upwelling of moist air from the $\sim 10$ bar level and below which breaks through the upper ammonia-ice cloud layer that subsequently darken with time over days as the disturbance subsides [Dyudina et al., 2007]. Their occurrence can also be monitored through $\sim \mathrm{MHz}$ radio bursts termed "Saturn electrostatic discharges" (SEDs), produced by (the generally unobserved) lightning strokes associated with the thunderstorm. Observations of SEDs and storm cloud features are essentially one-to-one when data on both are available [e.g., Kaiser et al., 1983; Fischer et al., 2008, $2011 \mathrm{a}, 2011 \mathrm{c}$. Unlike the NPH discussed above, however, thunderstorms do appear to be strongly seasonally controlled, being observed in the summer but not in the winter 
hemisphere (except immediately past equinox, as seen in section 5). This suggests that their occurrence is controlled through seasonal changes in the upper atmospheric layers that destabilize those below where sunlight does not penetrate.

[25] Thunderstorms were observed by Voyager in northern west jet $\mathrm{W}_{\mathrm{N}} 1\left(\sim 35^{\circ} \mathrm{N}\right)$ during northern spring conditions [Ingersoll et al., 1984]. They were also observed by Cassini in southern west jet $\mathrm{W}_{\mathrm{S}} 1\left(\sim 35^{\circ} \mathrm{S}\right)$ in southern summer and equinoctial conditions [Dyudina et al., 2007; Fischer et al., 2008, 2011a, 2011c], prior to the onset of the 2010-2011 GWS event in $W_{N} 1$ (see sections 4 and 5). The cyclonic (equatorward) and central portions of the west jets are thought generally to represent the locations of net rising air between the water cloud layer at depth and the upper ammonia-ice cloud layer in the slow meridional overturning circulation associated with the jet structure [Del Genio et al., 2009, Figure 6.18]. Since these storms do not greatly disturb the mean atmospheric zonal flow shown in Figure 1a, association with the west jets implies that storm rotation periods are close to System III, as first observed in Voyager data [Ingersoll et al., 1984]. Indeed, determinations of the zonal cloud velocities in the pre-equinox southern storms observed by Cassini in west jet $\mathrm{W}_{\mathrm{S}} 1$ indicate westward motions of typically only $\sim 3 \mathrm{~m} \mathrm{~s}^{-1}$ (typically $\sim 2-6 \mathrm{~m} \mathrm{~s}^{-1}$ ) relative to System III [Dyudina et al., 2007; Fischer et al., 2011a, $2011 \mathrm{c}]$. Their rotation periods are therefore slightly longer than System III, 10.659-10.664 h compared with $10.6562 \mathrm{~h}$ (Table 1) but a little shorter than the maximum period in west jet $\mathrm{W}_{\mathrm{S}} 1$ of $10.674 \mathrm{~h}$ (Table 2), the latter corresponding to a westward speed of $\sim 13 \mathrm{~m} \mathrm{~s}^{-1}$ relative to System III [e.g., García-Melendo et al., 2011]. The intervals and periods of southern storms A-I given by Fischer et al. [2011a, 2011c] are marked by the dark green lines labeled ST (southern thunderstorm) in Figures $2 \mathrm{~b}$ and $3 \mathrm{~b}$. As for the case of the NPH, the rotation periods of the southern thunderstorms are seen to be nearly independent of season (though their occurrence is not) and to be well separated from the PPO periods during southern summer. However, the northern PPO period is seen to converge toward similar periods across equinox and has closely similar periods for a short interval post equinox before the southern storms disappear in northern spring. The southern PPO period remained significantly longer than the thunderstorm periods throughout this interval.

\subsection{The "String of Pearls" Phenomenon}

[26] Contemporaneously with the intermittent southern thunderstorm activity observed by Cassini in $\mathrm{W}_{\mathrm{S}} 1$ during southern summer conditions, markedly different atmospheric features were observed at corresponding northern latitudes in $\mathrm{W}_{\mathrm{N}} 1$. These were initially discovered by Cassini VIMS as a chain of 20 long-lived IR-bright spots termed the string of pearls (SoP) [Momary et al., 2006; Choi et al., 2009], formed from low-opacity regions in the ammonium hydrosulfide cloud deck at $\sim 2-3$ bars that allowed observation of thermal emissions from below. They were also subsequently observed by Cassini ISS as low-contrast features in scattered sunlight after emerging from ring shadow in early 2007 [Sayanagi et al., 2013b]. These observations showed that the "pearls" form a chain of cyclonic vortices occupying roughly one quarter of the longitude range around the planet, centered in $\mathrm{W}_{\mathrm{N}} 1$ at $33.2^{\circ} \mathrm{N}$. They rotated westward with a speed of $22.27 \pm 0.20 \mathrm{~m} \mathrm{~s}^{-1}$ relative to System III over a measurement interval from September 2006 to December 2010 [Sayanagi et al., 2013b], corresponding to a rotation period of $10.6860 \pm 0.0003 \mathrm{~h}$ (Table 1). This is shown by the orange line labeled "SoP" in Figures $2 b$ and $3 b$, which is slightly longer than that of the corresponding west jet peak, with peak westward speeds of $\sim 20 \mathrm{~ms}^{-1}$. The SoP was eventually obliterated post equinox by the emergence of the GWS event in $\mathrm{W}_{\mathrm{N}} 1$ in December 2010, which formed centrally within the SoP structure, though the physical connection between them remains unclear. The phenomenon has not reemerged to date under northern spring conditions [Sayanagi et al., 2013b].

[27] The rotation period of the SoP, closely similar to that of the $\mathrm{W}_{\mathrm{N}} 1$ west jet peak in which it was located, is again comparable with those of the closely spaced PPO periods post equinox, specifically with the period of the southern PPOs under these conditions. In fact it can be seen from Figures $2 \mathrm{~b}$ and $3 \mathrm{~b}$ that in the same way that the northern PPO period converged post equinox on the period of the southern thunderstorms in $\mathrm{W}_{\mathrm{S}} 1$, the southern PPO period converged post equinox on the period of the SoP in $\mathrm{W}_{\mathrm{N}} 1$ (and that of the GWS that followed). Possible physical connections, however, remain equally obscure in both cases.

\subsection{Great White Spot Storms}

[28] The largest-scale phenomena observed in Saturn's atmosphere are the GWS events. These appear to be closely physically related to the thunderstorms discussed in section 3.2 but grow over intervals of days to much larger sizes, $\sim 10,000-20,000 \mathrm{~km}$, and produce atmospheric effects that persist for many months or even years (e.g., section 6.4.2 of the review by Del Genio et al. [2009]). They therefore affect the atmosphere over latitude bands $\sim 10^{\circ}-20^{\circ}$ and over increasing longitude ranges with time until after weeks the disturbances encircle the planet. One such event has been observed every Saturn year for the past six planetary years, thus at 230 year intervals, the first five occurring in post solstice northern summer conditions [Sánchez-Lavega, 1982, 1994; Beebe et al., 1992], while the most recent, in 2010-2011, was exceptional in occurring in northern spring [Sánchez-Lavega et al., 2011; Fischer et al., 2011b]. Alternate events have been initiated and centered in the northern part of the equatorial east jet, specifically those with onset in 1876, 1933, and 1990, while those between have been centered in one of the northern west jets, in $W_{N} 1$ in 1903 and 2010 and in $\mathrm{W}_{\mathrm{N}} 2$ in 1960. No counterpart is known in the Southern Hemisphere, during southern summer conditions, or otherwise.

[29] Because these events also do not hugely disturb the mean zonal flow, the rotation periods of the related clouds thus divide into two groups, depending on whether they are centered within the equatorial east jet or one of the northern west jets. Thus, the rotation period of the $1990 \mathrm{GWS}$, initiated in late September 1990 near $\sim 12^{\circ} \mathrm{N}$ planetocentric latitude in the northern part of the equatorial jet, which after expansion became centered near $\sim 4^{\circ} \mathrm{N}$, was found using ground-based and HST images to be $10.2412 \pm 0.0007 \mathrm{~h}$ (Table 1) over the interval between early October and midNovember 1990, thus characteristic of the equatorial jet [Beebe et al., 1992]. This period is shown in the central part of Figure 2b marked "1990 GWS," well-separated from the PPO periods of either hemisphere. Closely similar periods 
were also observed for the earlier equatorial GWS events, $10.242 \mathrm{~h}$ for the 1876 event centered at $\sim 7^{\circ} \mathrm{N}$ and $10.241 \mathrm{~h}$ for the 1933 event centered at $\sim 2^{\circ} \mathrm{N}$ [Sánchez-Lavega, 1982, 1994]. The periods of the GWS events occurring in the northern west jets in between the equatorial events, however, were significantly longer. The period of the 1903 event centered in $\mathrm{W}_{\mathrm{N}} 1$ at $\sim 31^{\circ} \mathrm{N}$ was $10.632 \mathrm{~h}$, while that of the 1960 event centered in $\mathrm{W}_{\mathrm{N}} 2$ at $\sim 52^{\circ} \mathrm{N}$ was $10.649 \mathrm{~h}$ [Sánchez-Lavega, 1982, 1994], thus reflecting the west jet periods. These values are then more akin to the PPO periods in the present epoch, specifically to the post equinox northern periods (Figures $2 \mathrm{~b}$ and $3 \mathrm{~b}$ ).

[30] Examining further the 1990 GWS event for which more detailed information is available, observations showed that following initial formation, the storm clouds became rapidly sheared by the zonal flow, to the west in the northern part of the cloud and to the east in the southern part, eventually encircling the planet, before degenerating by late December 1990 into an equatorial band of streaks and patches [SánchezLavega et al., 1991; Barnet et al., 1992]. Intermittent equatorial cloud activity then followed over the remaining years of northern summer, declining in 1997, 1 year after autumnal equinox [Sánchez-Lavega et al., 1993, 1999]. Notable among this activity was the appearance of a large "white spot" in summer 1994, $27,000 \mathrm{~km}$ in longitude by $\sim 12,000 \mathrm{~km}$ in latitude, centered near $\sim 7^{\circ} \mathrm{N}$, which persisted for more than a year. Ground-based observations between July 1994 and August 1995 indicate a rotation period of $10.366 \pm 0.004 \mathrm{~h}$ (Table 1), somewhat longer than the period of the mean zonal flow at this latitude ( 10.250 h) [Sánchez-Lavega et al., 1996, 1999]. This period is also shown in the central part of Figure $2 \mathrm{~b}$ marked "1994 WS," well-removed from any of the PPO periods measured at the same time by Ulysses. The equatorial GWS storms and related phenomena, occurring every other northern summer season to date, are thus the only large-scale atmospheric phenomena not associated with the west jets and rotating near the west jet periods, instead rotating with shorter periods characteristic of the equatorial jet where they occur. These GWS events therefore rotate with periods well-separated from the PPO periods, suggesting no essential connection between these phenomena.

\section{The 2010-2011 Great White Spot Storm}

[31] The GWS storm that erupted in December 2010 in early northern spring was centered in west jet $\mathrm{W}_{\mathrm{N}} 1$, as indicated in section 3 , and thus rotated near the west jet period like the earlier nonequatorial GWS events. The bright initial clouds of the GWS observed from the ground on 5 December 2010 grew over $\sim 10$ days to $\sim 10,000 \mathrm{~km}$ in size, spanning $\sim 10^{\circ}$ latitude centered near $\sim 34^{\circ} \mathrm{N}$ [Sánchez-Lavega et al., 2011; Fischer et al., 2011b; Sayanagi et al., 2013a]. Ground-based and Cassini ISS observations of the storm head, specifically of its westward leading edge, from its initiation in December 2010 to its extinction in June 2011, yielded a near-constant westward speed of $26.9 \pm 0.8 \mathrm{~m} \mathrm{~s}^{-1}$ relative to System III, corresponding to a rotation period of $10.693 \pm 0.001 \mathrm{~h}$ (Table 1 and Figures $2 \mathrm{~b}$ and $3 \mathrm{~b}$ ), thus being a little longer than that of the SoP and of the peak undisturbed period in $\mathrm{W}_{\mathrm{N}} 1$ [Sánchez-Lavega et al., 2012; Sayanagi et al., 2013a, 2013b]. This period is shown by the black line labeled "GWS-H" in Figure 2b, similarly lying close to the post equinox southern PPO period at that time.
[32] The relations for the 2010-2011 GWS are shown in greater detail in Figure 3b, where, as noted above, it is seen that the southern PPO period decreased to values close to that of the SoP and the peak of $\mathrm{W}_{\mathrm{N}} 1$ by mid-2010, several months before the eruption of the GWS, and then remained at such values during the initial interval of the storm. In midFebruary 2012, 2 months after the start of the storm, a small $\sim 0.2 \%$ increase in the southern PPO period then took place to values close to that of the slightly longer period of the GWS head (though not quite equal within uncertainty estimates), while simultaneously the northern PPO period decreased by $\sim 0.1 \%$ toward smaller values. The former jump in period corresponds to that previously noted by Fischer et al. [2012, 2013], though inferred to be of considerably larger amplitude $(\sim 0.5-1 \%)$ from their interpretation of SKR data. This sharp change, which also involved a sudden increase in southern PPO amplitude and a significant reduction in the northern amplitude [Provan et al., 2013], marked the start of the interval of closely separated PPO periods with abrupt $\sim 100-200$ day changes in properties that continues to the present (mid-2013). We thus note that the new behavior was initiated during the GWS event, but given the fact that further abrupt PPO changes of comparable nature have occurred subsequently (Figure 3b), well after the tropospheric storm had subsided, the significance of this temporal correspondence remains unclear. The closeness of the southern PPO period to that of the GWS head after the first jump also seems suggestive, but we note that the southern PPO period had approached such values several months earlier and has remained at similar values for nearly 3 years subsequently to the present. Bearing in mind the discussion in section 3.1 concerning coincidences in periods observed during the Voyager era, these data cannot be regarded as providing unambiguous indication of a physical connection between the PPOs and the GWS storm.

[33] Figure $3 \mathrm{~b}$ also provides further information concerning phenomena associated with the 2010-2011 GWS storm. Shortly after the appearance of the GWS bright head in early December 2010, a large dark anticyclonic vortex (AV) was formed to its east, which subsequently propagated eastward relative to the head at a speed of $\sim 19 \mathrm{~m} \mathrm{~s}^{-1}$, thus still moving westward in the System III frame at $\sim 8 \mathrm{~ms}^{-1}$ [Sánchez-Lavega et al., 2012; Sayanagi et al., 2013a]. The corresponding rotation period of this feature around the planet was $10.667 \pm 0.005 \mathrm{~h}$ (Table 1 ), shown by the black line marked "GWS-AV," lying centrally between the northern and southern PPO periods. Due to their relative motion, the main part of the storm system between the head and the AV expanded steadily in longitude over time, though we note that clouds at the northern and particularly the southern borders of the disturbance became more rapidly spread in the eastward direction due to the flow shear across the west jet, completely encircling the planet by mid-February 2011 [Sayanagi et al., 2013a]. However, by late June 2011 the AV had "lapped" the head, approaching from the west and coalescing with it, at which point the storm head collapsed over several days, leaving a disturbed band of clouds encircling the planet. The AV continues to exist to the time of writing (mid-2013), however, moving toward somewhat higher latitudes and shorter periods characteristic of the undisturbed wind profile, eventually off the scale of Figure 3b [Sayanagi et al., 2013a; K. M. Sayanagi, personal communication, 2013]. 
[34] In addition to these cloud-level disturbances, limited in altitude to the tropopause at $\sim 80$ mbar or a little above, the GWS storm also resulted in the formation of rotating "hot spots" in the stratosphere, observed by both Cassini CIRS and ground-based IR facilities, which have persisted to the end of presently available data at the end of 2012 [Fletcher et al., 2012; L. N. Fletcher, personal communication, 2013]. These perturbations, likely due to upward transmission of waves from the storm system, were observed between the tropopause and $\sim 0.1$ mbar, though with the largest increases in temperature, several tens of Kelvin, occurring between $\sim 5$ and $\sim 0.5$ mbar $(\sim 250-400 \mathrm{~km}$ above the 1 bar reference spheroid). At their first observation in January 2011, two such hot spots or "stratospheric beacons" were observed, one of them (here termed SB1) located over the GWS head and rotating with the same period, determined as $10.692 \pm 0.001 \mathrm{~h}$ by Fletcher et al. [2012], with the other (SB2) located to its east and rotating with a period shorter than the head and also somewhat shorter than the AV at cloud level, determined as $10.664 \pm 0.001 \mathrm{~h}$ (Table 1). These periods are shown by the purple lines and uncertainty estimate shading in Figure $3 \mathrm{~b}$. Due to their relative motion, these hot spots then met and coalesced at the end of April 2011, forming a single hot spot (SB0) that detached from the GWS head and rotated with an intermediate period of $10.677 \pm 0.003 \mathrm{~h}$, also shown in Figure $3 \mathrm{~b}$. With the disappearance of the tropospheric cloud head at the end of June 2011, the rotation period of SB0 then increased to $10.688 \pm 0.004 \mathrm{~h}$, equal within errors to the maximum undisturbed period in west jet $\mathrm{W}_{\mathrm{N}} 1$, and further to $10.696 \pm 0.001 \mathrm{~h}$ from late October 2011 to the end of 2012 (L. N. Fletcher, personal communication, 2013).

[35] These hot spots constitute anticyclonic flow vortices within the stratosphere that might possibly form rotating sources of perturbations in the upper atmosphere, due, e.g., to the excitation of upward propagating waves. The vortex associated with SB0, for example, was centered near $\sim 34^{\circ} \mathrm{N}$ and extended $\sim 10^{\circ}$ latitude on either side, with peak peripheral velocities of $\sim 200-400 \mathrm{~m} \mathrm{~s}^{-1}$ near 2 mbar as computed by Fletcher et al. [2012]. It can be seen from Figure 3b, however, that the planetary rotation periods of the hot spot, varying around the maximum undisturbed period in west jet $\mathrm{W}_{\mathrm{N}} 1$, do not agree at a detailed level with those determined for the southern PPO oscillations, within the uncertainties of both determinations. In particular, the changes in hot spot period are not contemporaneous with nor related in sense with those observed on similar time scales in the PPOs. Overall, while it is of interest to note the closeness of the rotation periods of these two phenomena in the post equinox interval, there nevertheless appears to be no consistent correspondence between them at a detailed level. Similarly to the NPS and thunderstorm phenomena discussed in sections 3.1 and 3.2 above, the atmospheric periods appear simply to be linked to those of the west jets, to which the PPOs for undetermined reasons then approximately correspond post equinox.

\section{Overall Correspondence Between PPOs and Thunderstorms During the Cassini Era}

[36] Overall correspondences between the PPOs and thunderstorm activity observed over the Cassini mission are explored more generally in Figure 4, where we plot relevant parameters versus time in days since the beginning of 2004 (the start of 1 January defines $t=0$ ). From top to bottom, we show (a) the rotation periods of the northern (blue) and southern (red) PPOs, (b) the ratio $k$ of the amplitudes of the northern and southern magnetic PPOs within the "core" magnetosphere (dipole $L \leq 12$ ), where $k$ is shown directly in the lower half of the panel and $1 / k$ in the upper half, thus spanning the full range of $k$ from zero (zero north amplitude) to infinity (zero south amplitude), (c) magnetic field oscillation phases of the three spherical polar field components measured on each Cassini Rev (marked with Rev number at periapsis at the top of the plot) shown in "southern format" relative to a guide phase corresponding to a fixed period of $10.69 \mathrm{~h}$, from which the southern phase is obtained versus time (black line), and (d) the magnetic field oscillation phases shown in "northern format" relative to a guide phase corresponding to a fixed period of $10.64 \mathrm{~h}$, from which the northern phase is obtained versus time (black line). Start-of-year boundaries are shown at the top of the figure, together with vernal equinox (vertical dotted line) and an interval identifier where blue corresponds to near-equatorial Cassini orbits and green to polar, thus yielding differing types of magnetic field PPO data. These data originate from Andrews et al. [2012] and Provan et al. [2013], the latter being updated to mid-2013, from which further detail can be obtained (see also the figure caption). The lettered pink bands then show the times of southern thunderstorm activity, all centered in west jet $\mathrm{W}_{\mathrm{S}} 1$ at $\sim 35^{\circ} \mathrm{S}$, storms $\mathrm{A}-\mathrm{C}$ were those analyzed by Dyudina et al. [2007], while storms A-I were included in the studies by Fischer et al. [2011a, 2011c], for which the rotation periods are shown in Figures $2 \mathrm{~b}$ and $3 \mathrm{~b}$ and given in Table 1. The blue band corresponds to the 2010-2011 GWS event centered in west jet $\mathrm{W}_{\mathrm{N}} 1$ at $\sim 34^{\circ} \mathrm{N}$ [Sánchez-Lavega et al., 2011; Fischer et al., 2011b; Sayanagi et al., 2013a], representing the first northern thunderstorm activity post equinox. Following the GWS, there has been no evidence of long-lasting thunderstorm features in available Cassini ISS images to the time of writing (K. M. Sayanagi, personal communication, 2013).

[37] Figure 4 firstly indicates that the switch in storm location from Southern to Northern Hemisphere occurred between the end of storm I in late June 2010 and the beginning of the GWS in early December 2010, thus approximately 1 year after equinox, the latter marked by the vertical dotted line. This transition was thus centered on the near-coalescence of the north and south PPO periods in mid-September 2010 shown in Figure $4 \mathrm{a}$ (see also Figure $3 \mathrm{~b}$ ), though again the physical significance of this temporal correspondence, if any, is unknown. The subsequent transition in behavior of the PPOs initiated during the GWS event noted above, from properties that vary relatively smoothly with time to abrupt Rev-to-Rev changes at $\sim 100-200$ day intervals, is also very clear in the phase data in Figures $4 \mathrm{c}$ and $4 \mathrm{~d}$, as marked by the vertical dashed lines. It is also evident, however, that the temporal correspondences are by no means exact. As noted in section 4, the first transition at the end of interval E1 with near-equal PPO amplitudes $(k \approx 1)$ and closely spaced periods, to interval E2 with unexpectedly resumed southern dominance $(k \approx 0.3)$ and somewhat more separated periods, occurred in mid-February 2011, more than 2 months after the start of the GWS event. Similarly, the subsequent transition to interval E3 with strong northern dominance $(k \approx 6)$ occurred in mid-August 2011, nearly 2 months after the subsidence of the storm in late June, and after the main decline in SEDs, though sporadic SED activity persisted at least until mid-July [Sayanagi et al., 2013a]. We also note that 
COWLEY AND PROVAN: MAGNETOSPHERIC OSCILLATIONS AND THE GWS

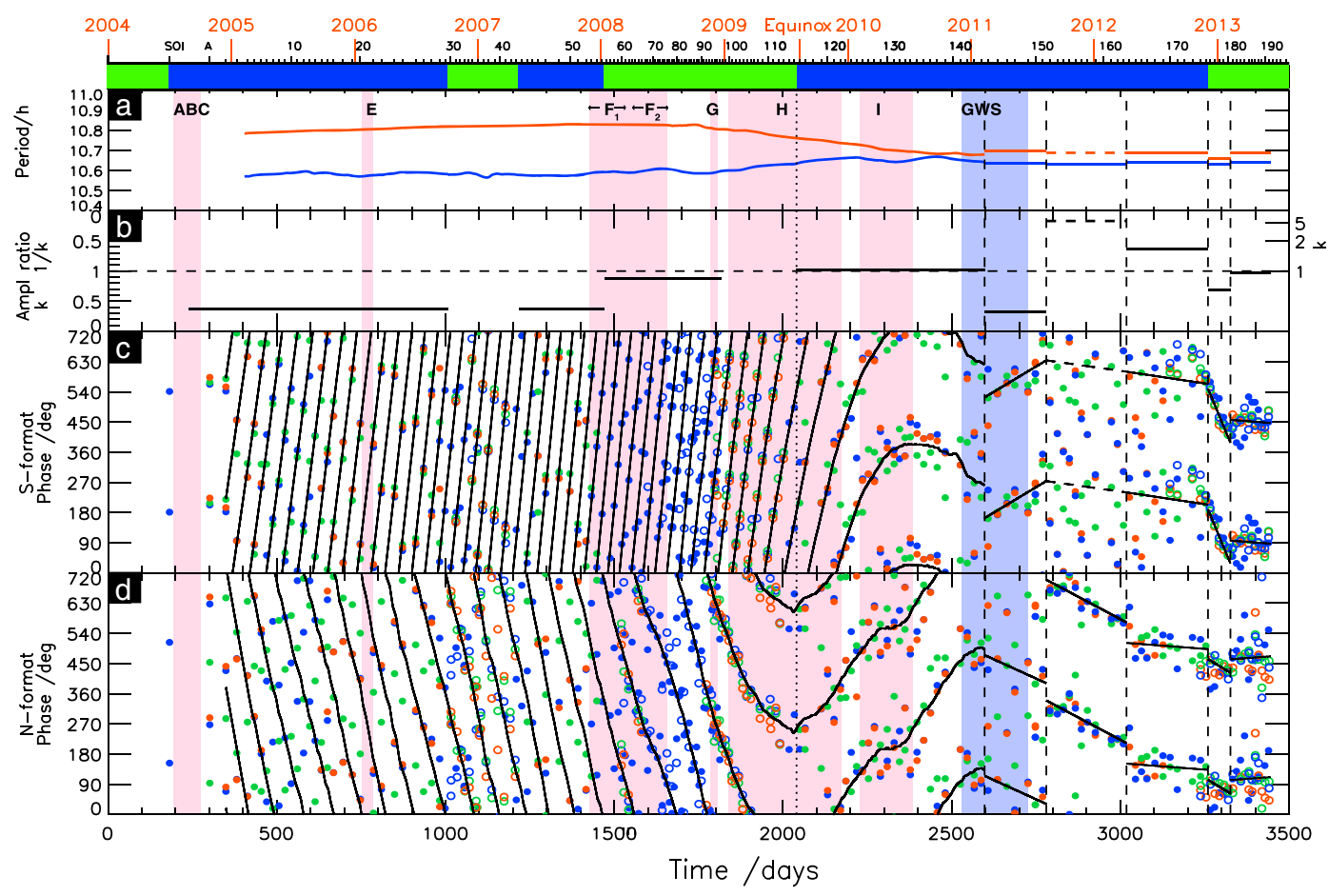

Figure 4. Plot showing the temporal evolution of PPO properties over the Cassini mission to date in relation to thunderstorm activity, plotted versus time in days since the beginning of 1 January 2004. Start-of-year boundaries are shown at the top of the figure, as are the periapsis times of Cassini marked with their Rev number every tenth Rev, and an orbit interval identifier following the schemes of Andrews et al. [2012] and Provan et al. [2013]. Dark blue intervals indicate near-equatorial Cassini orbits principally yielding "core" magnetic oscillation phase data on closed magnetospheric field lines, while green indicates polar orbits principally yielding magnetic phase data on open magnetospheric field lines. The time of vernal equinox is shown by the vertical dotted line as also indicated at the top of the plot. (a) The rotation periods of the northern (blue) and southern (red) PPOs derived from Cassini magnetic field data. (b) The ratio $k$ of the amplitudes of the north divided by the south magnetic field PPOs within the core magnetosphere (dipole $L \leq 12$ ), where $k$ is shown directly in the lower half of the panel and $1 / k$ in the upper half, with the dashed line at $k=1$ indicating equal amplitudes. (c) The magnetic field oscillation phases of the three field components within the core region (solid circles) and on polar field lines (open circles) measured on each Cassini Rev, red for the $r$ component, green for $\theta$, and blue for $\phi$, shown in "southern format" relative to a guide phase corresponding to a fixed period of $10.69 \mathrm{~h}$, from which the relative southern phase is obtained versus time shown by the black line. Two cycles of phase are shown on the vertical axis to better exhibit the continuity of the data, with each point thus being plotted just twice. (d) The magnetic field oscillation phases in "northern format" relative to a guide phase corresponding to a fixed period of $10.64 \mathrm{~h}$, from which the northern phase is obtained versus time, also shown by the black line. The data in panels Figures 4a-4d were obtained from Andrews et al. [2012] and Provan et al. [2013], updated in the latter case to mid-2013. The lettered pink bands show the times of southern thunderstorm activity in west jet $\mathrm{W}_{\mathrm{S}} 1$ following the studies of Dyudina et al. [2007] and Fischer et al. [2011a, 2011c], while the blue band corresponds to the 2010-2011 GWS event centered in west jet $\mathrm{W}_{\mathrm{N}} 1$ [SánchezLavega et al., 2011; Fischer et al., 2011b; Sayanagi et al., 2013a]. No reports of major thunderstorm activity following the GWS have been made to date.

the transitions between intervals E3, E4, F1, and F2 all appear to have occurred in the absence of major thunderstorm activity, with the transition from E4 to F1, in particular, having a number of similar characteristics to that occurring during the GWS. Careful examination of the behavior of the PPO phases during the earlier southern storms using guide periods that are more appropriate to those intervals has also failed to reveal any clear related effects, though of course these storms disturbed the atmosphere to a much smaller extent than did the GWS.

[38] Overall, this comparison reveals a number of features that occur near-contemporaneously in the seasonal evolution of both the PPO and thunderstorm behavior, specifically the convergence in PPO periods post equinox close to the time when storm activity switched from Southern to Northern Hemispheres, and the change in PPO behavior from relatively smoothly varying to abrupt $\sim 100-200$ day transitions that occurred $\sim 2$ months after the start of the GWS event. While these correspondences may be connected at some general level, due, e.g., to ongoing seasonally related effects, the lack of clear and consistent correspondence between these phenomena does not suggest a connection at a detailed level. 


\section{Discussion and Summary}

[39] We have made a first exploratory investigation of the empirical relations between the rotation periods of the PPO perturbations in Saturn's magnetosphere and the rotation periods of Saturn's neutral atmosphere and its associated phenomena. The study has been motivated by the suggestion that the magnetospheric PPOs could be driven by a rotating twin-vortical wind system in the thermosphere [Smith, 2006, 2010; Jia et al., 2012; Jia and Kivelson, 2012], which might in turn be driven by a rotating source of momentum in the lower atmosphere (though other possibilities also exist). In particular, Fischer et al. [2012, 2013] have suggested that the PPO period, specifically that of the southern system, was affected by the occurrence of the 2010-2011 northern GWS thunderstorm event. It is in any case evident that the PPO periods must be maintained in some manner by the internal rotation of the planet, coupled through the lower and middle atmospheric layers, such that comparisons with atmospheric-related rotation periods are relevant to issues of origin.

[40] Winds in Saturn's upper troposphere determined from cloud motions have been found to exhibit a quite wide range of rotation periods associated with the zonal jet structure, from lowest values of $\sim 10.25 \mathrm{~h}$ in the central equatorial east jet to $\sim 10.35-10.45 \mathrm{~h}$ in the peaks of the off-equatorial east jets and $\sim 10.60-10.70 \mathrm{~h}$ in the peaks of the off-equatorial west jets [e.g., Ingersoll et al., 1984; García-Melendo et al., 2011]. This jet structure is also likely to remain intact within the stratosphere to $\sim 300 \mathrm{~km}$ altitudes and possibly beyond [Fouchet et al., 2008; Read et al., 2009b; Fletcher et al., 2012]. The rotation periods of the PPOs, varying over the range $\sim 10.57-10.66 \mathrm{~h}$ for the northern system and $\sim 10.66-10.83 \mathrm{~h}$ for the southern over the Cassini mission to date, are then most closely similar to those of the west jet peaks, remaining significantly longer than the periods of the peaks of the east and equatorial jets.

[41] The most important difference between the rotation periods of the neutral atmosphere and the PPOs is that the neutral atmospheric periods are nearly independent of Saturn's seasons, while the PPO periods undergo a seasonal cycle. Specifically, the northern and southern PPO periods were well-separated during southern summer, $\sim 10.57-10.59 \mathrm{~h}$ for the northern system and $\sim 10.77-10.83 \mathrm{~h}$ for the southern [e.g., Andrews et al., 2012]. The northern period thus lay between those of the east and west jets close to the deep rotation period of the planet estimated by Read et al. [2009a], while the southern period was longer than any associated with lower atmospheric rotation periods, thus implying that it was not driven in any direct way from the lower layers. The PPO periods then converged across equinox to more closely spaced values of $\sim 10.63-10.66 \mathrm{~h}$ for the northern system and 10.66-10.69 h for the southern [e.g., Provan et al., 2013], thus lying at values directly comparable with the west jet periods. The physical significance of these empirical relations, if any at a detailed level, remains unclear.

[42] This empirical relation between the equinoctial PPO periods and those of the west jets is, in effect, already recognized in the wind speeds measured in the standard System III reference frame (e.g., Figure 1). This frame is keyed to the period of the dominant SKR modulations observed during the Voyager 1 encounter, taken at that time to correspond to the deep rotation period of the planet. Because the flyby occurred shortly after Saturn vernal equinox, such that the dominant north PPO period was $\sim 10.66 \mathrm{~h}$, close to that of the west jet peaks, the west jet wind speeds in this frame peak close to zero. If the flyby had instead occurred, e.g., during southern solstice when the southern PPO period was dominant at $\sim 10.8 \mathrm{~h}$, the atmospheric winds in the PPO frame would all have been strongly "supercorotational." Such a result might have provided an early indication that all was not as it seemed, particularly given the somewhat more "balanced" nature of the periods of Jupiter's atmospheric east and west jets about the well-determined planetary rotation period in that case [e.g., Ingersoll et al., 2004].

[43] A further empirical fact is that most of the large-scale atmospheric phenomena observed in Saturn's atmosphere are related to the west jets and rotate with closely similar periods. This applies to the seasonal thunderstorm activity that occurs within the summer hemisphere, to the SoP phenomenon, and to every other GWS northern storm (1903, 1960, and 2010) observed to date. Even the NPH feature, though located in east jet $\mathrm{E}_{\mathrm{N}} 3$, rotates together with its erstwhile NPS companion with a period related to adjacent west jet $\mathrm{W}_{\mathrm{N}} 3$. As a consequence, these atmospheric phenomena also rotate with periods that are closely comparable with the PPO periods under equinoctial conditions (but not otherwise). Inferences drawn concerning physical connections based on close commonality of rotation periods under such conditions need therefore to be carefully considered and may prove to be unfounded when the overall picture is taken into account. A specific instance is the close correspondence observed between the NPH/NPS and northern PPO rotation periods under post equinoctial conditions in Voyager flyby data, within tight uncertainty limits, which has later proven to be unsustained under southern summer conditions in Cassini data.

[44] With regard to seasonal thunderstorm activity in the Cassini era, we have found empirically that the northern PPOs converge post equinox to a period close to that of the southern summer and equinoctial storms in $\mathrm{W}_{\mathrm{S}} 1$, while the southern PPOs converge simultaneously to a slightly longer period close to that of the northern SoPs and 2010-2011 GWS in $\mathrm{W}_{\mathrm{N}} 1$. The physical significance of these curiously coincident values, if any in detail, also remains unclear (also noting that the previous off-equatorial GWS events in 1903 and 1960 had slightly shorter periods closer to the post equinox northern PPOs in the present era). We have also found that the seasonal change from southern to northern storms occurred near-contemporaneously with the closest convergence of the PPO periods in mid-September 2010, about 1 year after equinox, and that the PPO regime with closely spaced periods and abrupt $\sim 100-200$ day jumps in properties was initiated during the northern GWS thunderstorm that followed. Again, the physical significance of these coincident events, if any beyond essentially independent responses e.g. to the changing seasons or solar activity, remains unclear.

[45] Overall, our discussion suggests that considerable caution should be exercised when drawing inferences of physical significance from correspondences between the rotation periods of the southern PPOs and those associated with the GWS storm, in particular the jump in southern PPO period to a value close to that of the storm head that occurred in mid-February 2011 pointed out by Fischer et al. [2012, 2013]. It should be emphasized, however, that the southern PPO had been close to this period for several 
months before the onset of the GWS that the jumps to and from this period are far from contemporaneous with the start and end of the main tropospheric activity and that a number of similar abrupt PPO changes have occurred subsequently without benefit of related major thunderstorm activity. Conversely, pre-equinox thunderstorm activity has been found to produce no discernible contemporaneous effect on the PPOs. Hot spots generated by the GWS have remained present in the stratosphere throughout this interval, but while again rotating with a period close to that of the southern PPOs, no clear correspondences have been found at a detailed level between the variations in their rotation period and those of the southern PPOs. We further note that every other GWS event, like that in 1990, has been observed to occur within the northern equatorial eastward jet, then rotating with much shorter periods than any associated with the PPOs, thus indicating that no essential connection exists. Overall, these results do not convincingly suggest that a direct physical link exists between the PPOs and thunderstorm activity, GWS-related or otherwise, such as would be expected if, e.g., they were directly driven or significantly influenced by upward propagation of $m=1$ perturbations from such lower atmospheric storm systems.

[46] Acknowledgments. This work was supported by STFC grant ST/ K001000/1. We thank K.H. Baines for helpful comments on Cassini/VIMS observations of the north polar hexagon, L.N. Fletcher for updated information on the GWS-related stratospheric beacons based on Cassini/CIRS and ground data employed in Figure 3, E. García-Melendo for the Cassini/ISS tropospheric wind data employed in Figure 1, D.P. Milika and P. Nicholas for access to small-telescope Saturn images, and K.M. Sayanagi for many helpful comments concerning GWS, SoP, and thunderstorm activity observed by Cassini/ISS.

[47] Masaki Fujimoto thanks the reviewers for their assistance in evaluating this paper.

\section{References}

Andrews, D. J., E. J. Bunce, S. W. H. Cowley, M. K. Dougherty, G. Provan, and D. J. Southwood (2008), Planetary period oscillations in Saturn's magnetosphere: Phase relation of equatorial magnetic field oscillations and SKR modulation, J. Geophys. Res., 113, A09205, doi:10.1029/ 2007JA012937.

Andrews, D. J., S. W. H. Cowley, M. K. Dougherty, and G. Provan (2010a), Magnetic field oscillations near the planetary period in Saturn's equatorial magnetosphere: Variation of amplitude and phase with radial distance and local time, J. Geophys. Res., 115, A04212, doi:10.1029/2007JA014729.

Andrews, D. J., A. J. Coates, S. W. H. Cowley, M. K. Dougherty, L. Lamy, G. Provan, and P. Zarka (2010b), Magnetospheric period oscillations at Saturn: Comparison of equatorial and high-latitude magnetic field periods with north and south SKR periods, J. Geophys. Res., 115, A12252, doi:10.1029/2010JA015666.

Andrews, D. J., B. Cecconi, S. W. H. Cowley, M. K. Dougherty, L. Lamy, G. Provan, and P. Zarka (2011), Planetary period oscillations in Saturn's magnetosphere: Evidence in magnetic field phase data for rotational modulation of Saturn kilometric radiation emissions, J. Geophys. Res., 116, A09206, doi:10.1029/2011JA016636.

Andrews, D. J., S. W. H. Cowley, M. K. Dougherty, L. Lamy, G. Provan, and D. J. Southwood (2012), Planetary period oscillations in Saturn's magnetosphere: Evolution of magnetic oscillation properties from southern summer to post-equinox, J. Geophys. Res., 117, A04224, doi:10.1029/2011JA017444.

Arridge, C. S., et al. (2011), Upstream of Saturn and Titan, Space Sci. Rev., $162,25-83$

Badman, S. V., S. W. H. Cowley, B. Cecconi, L. Lamy, and P. Zarka (2008), Relationship between solar wind corotating interaction regions and the phasing and intensity of Saturn kilometric radiation bursts, Ann. Geophys., 26, 3641-3651.

Badman, S. V., et al. (2012), Rotational modulation and local time dependence of Saturn's infrared $\mathrm{H}_{3}{ }^{+}$auroral intensity, J. Geophys. Res., 117, A09228, doi:10.1029/2011JA017990.

Baines, K. H., T. W. Momary, L. Fletcher, B. J. Buratti, M. Roose-Serote, A. Showman, R. H. Brown, R. N. Clark, and P. D. Nicholson (2008),
Saturn's north polar vortex revealed by Cassini/VIMS: Zonal wind structure and constraints on cloud distributions, Bull. Am. Astron. Soc., 40, 495 .

Baines, K. H., T. W. Momary, L. N. Fletcher, A. P. Showman, M. Roose-Serote, R. H. Brown, B. J. Buratti, R. N. Clark, and P. D. Nicholson (2009), Saturn's north polar cyclone and hexagon at depth revealed by Cassini/VIMS, Planet. Space Sci., 57, 1671-1681.

Barnet, C. D., J. A. Westphal, R. F. Beebe, and L. F. Huber (1992), Hubble Space Telescope observations of the 1990 equatorial disturbance on Saturn: Zonal winds and central meridian albedos, Icarus, 100, 499-511.

Beebe, R. F., C. Barnet, P. V. Sada, and A. S. Murrell (1992), The onset and growth of the 1990 equatorial disturbance on Saturn, Icarus, 95, 163-172.

Brandt, P. C., K. K. Khurana, D. G. Mitchell, N. Sergis, K. Dialynas, J. F. Carbary, E. C. Roelof, C. P. Paranicas, S. M. Krimigis, and B. H. Mauk (2010), Saturn's periodic magnetic field perturbations caused by a rotating partial ring current, Geophys. Res. Lett., 37, L22103, doi:10.1029/2010GL045285

Burch, J. L., A. D. DeJong, J. Goldstein, and D. T. Young (2009), Periodicity in Saturn's magnetosphere: Plasma cam, Geophys. Res. Lett., 36, L14203, doi:10.1029/2009GL039043.

Burton, M. E., M. K. Dougherty, and C. T. Russell (2010), Saturn's internal planetary magnetic field, Geophys. Res. Lett., 37, L24105, doi:10.1029/ 2010 GL045148.

Carbary, J. F. (2013), Longitude dependence of Saturn's ultraviolet aurora, Geophys. Res. Lett., 40, 1902-1906, doi:10.1002/grl.50430.

Carbary, J. F., and S. M. Krimigis (1982), Charged particle periodicity in the Saturnian magnetosphere, Geophys. Res. Lett., 9, 1073-1076.

Carbary, J. F., D. G. Mitchell, S. M. Krimigis, and N. Krupp (2007), Electron periodicities in Saturn's outer magnetosphere, J. Geophys. Res., 112, A03206, doi:10.1029/2006JA012077.

Carbary, J. F., D. G. Mitchell, P. Brandt, C. Paranicas, and S. M. Krimigis (2008a), ENA periodicities at Saturn, Geophys. Res. Lett., 35, L07102, doi:10.1029/2008GL033230.

Carbary, J. F., D. G. Mitchell, P. Brandt, E. C. Roelof, and S. M. Krimigis (2008b), Periodic tilting of Saturn's plasma sheet, Geophys. Res. Lett., 35, L24101, doi:10.1029/2008GL036339.

Choi, D. S., A. P. Showman, and R. H. Brown (2009), Cloud features and zonal wind measurements of Saturn's atmosphere as observed by Cassini/VIMS, J. Geophys. Res., 114, E04007, doi:10.1029/ 2008JE003254.

Clarke, K. E., et al. (2006), Cassini observations of planetary-period oscillations of Saturn's magnetopause, Geophys. Res. Lett., 33, L23104, doi:10.1029/2006GL027821.

Clarke, K. E., D. J. Andrews, A. J. Coates, and S. W. H. Cowley (2010a), Magnetopause oscillations near the planetary period at Saturn: Occurrence, phase and amplitude, J. Geophys. Res., 115, A08209, doi:10.1029/2009JA014745.

Clarke, K. E., D. J. Andrews, A. J. Coates, S. W. H. Cowley and A. Masters (2010b), Magnetospheric period oscillations of Saturn's bow shock, J. Geophys. Res., 115, A05202, doi:10.1029/2009JA015164.

Cowley, S. W. H., S. V. Badman, E. J. Bunce, J. T. Clarke, J.-C. Gérard, D. Grodent, C. M. Jackman, S. E. Milan, and T. K. Yeoman (2005), Reconnection in a rotation-dominated magnetosphere and its relation to Saturn's auroral dynamics, J. Geophys. Res., 110, A02201, doi:10.1029/ 2004JA010796.

Cowley, S. W. H., D. M. Wright, E. J. Bunce, A. C. Carter, M. K. Dougherty, G. Giampieri, J. D. Nichols, and T. R. Robinson (2006), Cassini observations of planetary- period magnetic field oscillations in Saturn's magnetosphere: Doppler shifts and phase motion, Geophys. Res. Lett., 33, L07104, doi:10.1029/2005GL025522.

Davies, M. E., V. K. Abalakin, J. H. Lieske, P. K. Seidelmann, A. T. Sinclair, A. M. Sinzi, B. A. Smith, and Y. S. Tjuflin (1983), Report of the IAU Working Group on cartographic coordinates and rotational elements of the planets and satellites: 1982, Celestial Mech., 29, 309-321.

Del Genio, A. D., R. K. Achterberg, K. H. Baines, F. M. Flasar, P. L. Read, A. Sánchez-Lavega, and A. P. Showman (2009), Saturn atmospheric structure and dynamics, in Saturn From Cassini-Huygens, edited by M. K. Dougherty, L. W. Esposito, and S. M. Krimigis, pp. 113-159, Springer Publ., Netherlands.

Desch, M. D., and M. L. Kaiser (1981), Voyager measurement of the rotation period of Saturn's magnetic field, Geophys. Res. Lett., 8, 253-256.

Dyudina, U. A., A. P. Ingersoll, S. P. Ewald, C. C. Porco, G. Fischer, W. Kurth, M. Desch, A. Del Genio, J. Barbara, and J. Ferrier (2007), Lightning storms on Saturn observed by Cassini ISS and RPWS during 2004-2006, Icarus, 190, 545-555.

Espinosa, S. A., and M. K. Dougherty (2000), Periodic perturbations in Saturn's magnetic field, Geophys. Res. Lett., 27, 2785-2788.

Fischer, G., D. A. Gurnett, W. S. Kurth, F. Akalin, P. Zarka, U. A. Dyudina, W. M. Farrell, and M. L. Kaiser (2008), Atmospheric electricity at Saturn, Space Sci. Rev., 137, 271-285. 
Fischer, G., D. A. Gurnett, P. Zarka, L. Moore, and U. A. Dyudina (2011a), Peak electron densities in Saturn's ionosphere derived from low-frequency cutoff of Saturn lightning, J. Geophys. Res., 116, A04315, doi:10.1029/2010JA016187.

Fischer, G., et al. (2011b), A giant thunderstorm on Saturn, Nature, 475, 75-77.

Fischer, G., et al. (2011c), Overview of Saturn lightning observations, in Planetary Radio Emissions VII, edited by H. O. Rucker et al., pp. 135-144, Austrian Acad. Sci. Press, Vienna.

Fischer, G., D. A. Gurnett, S.-Y. Ye, J. B. Groene, J. D. Menietti, and W. S. Kurth (2012), A possible influence of the Great White Spot on the rotation of Saturn's magnetosphere, EPSC Abstracts, 7, EPSC2012-33, 2012.

Fischer, G., D. A. Gurnett, S.-Y. Ye, J. B. Groene, A. P. Ingersoll, K. M. Sayanagi, J. D. Menietti, and W. S. Kurth (2013), Saturn's magnetospheric rotation after equinox and a possible influence by the Great White Spot, EPSC Abstracts, 8, EPSC2013-1011, 2013.

Fletcher, L. N., et al. (2008), Temperature and composition of Saturn's polar hot spots and hexagon, Science, 319, 79-81.

Fletcher, L. N., et al. (2012), The origin and evolution of Saturn's 2011-2012 stratospheric vortex, Icarus, 221, 560-586.

Fouchet, T., S. Guerlet, D. F. Strobel, A. A. Simon-Miller, B. Bézard, and F. M. Flasar (2008), An equatorial oscillation in Saturn's middle atmosphere, Nature, 453, 200-202.

Galand, M., L. Moore, I. Mueller-Wodarg, M. Mendillo, and S. Miller (2011), Response of Saturn's auroral ionosphere to electron precipitation: Electron density, electron temperature, and electrical conductivity, J. Geophys. Res., 116, A09306, doi:10.1029/2010JA016412.

Galopeau, P. H. M., and A. Lecacheux (2000), Variations of Saturn's radio rotation period measured at kilometer wavelengths, J. Geophys. Res., 105, 13,089-13,101.

García-Melendo, E., S. Péres-Hoyos, A. Sánchez-Lavega, and R. Hueso (2011), Saturn's zonal wind profile in 2004-2009 from Cassini ISS images and its long-term variability, Icarus, 215, 62-74.

Godfrey, D. A. (1988), A hexagonal feature around Saturn's north pole, Icarus, 76, 335-356.

Godfrey, D. A. (1990), The rotation period of Saturn's polar hexagon, Science, 247, 1206-1208.

Gurnett, D. A., W. S. Kurth, and F. L. Scarf (1981), Plasma waves near Saturn: Initial results from Voyager 1, Science, 212, 235-239.

Gurnett, D. A., et al. (2005), Radio and plasma wave observations at Saturn from Cassini's approach and first orbit, Science, 307, 1255-1259.

Gurnett, D. A., A. M. Persoon, W. S. Kurth, J. B. Groene, T. F. Averkamp, M. K. Dougherty, and D. J. Southwood (2007), The variable rotation period of the inner region of Saturn's plasma disk, Science, 316, 442-445, doi:10.1126/science.1138562.

Gurnett, D. A., A. Lecacheux, W. S. Kurth, A. M. Persoon, J. B. Groene, L. Lamy, P. Zarka, and J. F. Carbary (2009a), Discovery of a north-south asymmetry in Saturn's radio rotation period, Geophys. Res. Lett., 36, L16102, doi:10.1029/2009GL039621.

Gurnett, D. A., A. M. Persoon, J. B. Groene, A. J. Kopf, G. B. Hospodarsky, and W. S. Kurth (2009b), A north-south difference in the rotation rate of auroral hiss at Saturn: Comparison to Saturn's kilometric radio emission, Geophys. Res. Lett., 36, L21108, doi:10.1029/2009GL040774.

Gurnett, D. A., et al. (2010a), A plasmapause-like density boundary at high latitudes in Saturn's magnetosphere, Geophys. Res. Lett., 37, L16806, doi:10.1029/2010GL044466.

Gurnett, D. A., J. B. Groene, A. M. Persoon, J. D. Menietti, S.-Y. Ye, W. S. Kurth, R. J. MacDowell, and A. Lecacheux (2010b), The reversal of the rotational modulation rates of the north and south components of Saturn kilometric radiation near equinox, Geophys. Res. Lett., 37, L24101, doi:10.1029/2010GL045796.

Gurnett, D. A., J. B. Groene, T. F. Averkamp, W. S. Kurth, S.-Y. Ye, and G. Fischer (2011), The SLS4 longitude system based on a tracking filter analysis of the rotational modulation of Saturn kilometric radiation, in Planetary Radio Emissions VII, edited by H. O. Rucker et al., pp. 51-64, Austrian Acad. Sci. Press, Vienna.

Ingersoll, A. P., R. F. Beebe, B. J. Conrath, and G. E. Hunt (1984), Structure and dynamics of Saturn's atmosphere, in Saturn, edited by T. Gehrels and M. S. Matthews, pp. 195-238, Univ. Arizona Press, Tucson.

Ingersoll, A. P., T. E. Dowling, P. J. Gierasch, G. S. Orton, P. L. Read, A. Sánchez-Lavega, A. P. Showman, A. A. Simon-Miller, and A. R. Vasavada (2004), Dynamics of Jupiter's atmosphere, in Jupiter: The Planet, Satellites and Magnetosphere, edited by F. Bagenal, T. E. Dowling, and W. B. McKinnon, pp. 105-128, Cambridge Univ. Press, Cambridge, U.K

Jia, X., and M. G. Kivelson (2012), Driving Saturn's magnetospheric periodicities from the upper atmosphere/ionosphere: Magnetotail response to dual sources, J. Geophys. Res., 117, A11219, doi:10.1029/2012JA018183.

Kaiser, M. L., J. E. P. Connerney, and M. D. Desch (1983), Atmospheric storm explanation of Saturnian electrostatic discharges, Nature, 303, $50-53$.
Kurth, W. S., A. Lecacheux, T. F. Averkamp, J. B. Groene, and D. A. Gurnett (2007), A Saturnian longitude system based on a variable kilometric radiation period, Geophys. Res. Lett., 34, L02201, doi:10.1029/2006GL028336.

Kurth, W. S., T. F. Averkamp, D. A. Gurnett, J. B. Groene, and A. Lecacheux (2008), An update to a Saturnian longitude system based on kilometric radio emissions, J. Geophys. Res., 113, A05222, doi:10.1029/2007JA012861.

Lamy, L. (2011), Variability of southern and northern SKR periodicities, in Planetary Radio Emissions VII, edited by H. O. Rucker et al., pp. 39-50, Austrian Acad. Sci. Press, Vienna, Austria.

Matcheva, K. I., and D. J. Barrow (2012), Small-scale variability in Saturn's lower ionosphere, Icarus, 221, 525-543.

Momary, T. W., K. H. Baines, and the Cassini/VIMS Science Team (2006), The zoology of Saturn: The bizarre features unveiled by the 5 micron eyes of Cassini/VIMS, Bull. Am. Astron. Soc., 38, 499.

Nichols, J. D., J. T. Clarke, S. W. H. Cowley, J. Duval, A. J. Farmer, J.-C. Gérard, D. Grodent, and S. Wannawichian (2008), Oscillation of Saturn's southern auroral oval, J. Geophys. Res., 113, A11205, doi:10.1029/2008JA013444.

Nichols, J. D., B. Cecconi, J. T. Clarke, S. W. H. Cowley, J.-C. Gérard, A. Grocott, D. Grodent, L. Lamy, and P. Zarka (2010a), Variation of Saturn's UV aurora with SKR phase, Geophys. Res. Lett., 37, L15102, doi:10.1029/2010GL044057.

Nichols, J. D., S. W. H. Cowley, and L. Lamy (2010b), Dawn-dusk oscillation of Saturn's conjugate auroral ovals, Geophys. Res. Lett., 37, L24102, doi:10.1029/2010GL045818.

Provan, G., D. J. Andrews, C. S. Arridge, S. W. H. Cowley, S. E. Milan, M. K. Dougherty, and D. M. Wright (2009a), Polarization and phase of planetary period oscillations on high latitude field lines in Saturn's magnetosphere, J. Geophys. Res., 114, A02225, doi:10.1029/2008JA013782.

Provan, G., S. W. H. Cowley, and J. D. Nichols (2009b), Phase relation of oscillations near the planetary period of Saturn's auroral oval and the equatorial magnetospheric magnetic field, J. Geophys. Res., 114, A04205, doi:10.1029/2008JA013988.

Provan, G., D. J. Andrews, B. Cecconi, S. W. H. Cowley, M. K. Dougherty, L. Lamy, and P. Zarka (2011), Magnetospheric period magnetic field oscillations at Saturn: Equatorial phase "jitter" produced by superposition of southern and northern period oscillations, J. Geophys. Res., 116, A04225, doi:10.1029/2010JA016213.

Provan, G., D. J. Andrews, C. S. Arridge, A. J. Coates, S. W. H. Cowley, G. Cox, M. K. Dougherty, and C. M. Jackman (2012), Dual periodicities in planetaryperiod magnetic field oscillations in Saturn's tail, J. Geophys. Res., 117, A01209, doi:10.1029/2011JA017104.

Provan, G., S. W. H. Cowley, J. Sandhu, D. J. Andrews, and M. K. Dougherty (2013), Planetary period magnetic field oscillations in Saturn's magnetosphere: Post-equinox abrupt non-monotonic transitions to northern system dominance, J. Geophys. Res. Space Physics, 118, 3243-3264, doi:10.1002/jgra.50186.

Read, P. L., T. E. Dowling, and G. Schubert (2009a), Saturn's rotation period from its atmospheric planetary-wave configuration, Nature, 460, 608-610.

Read, P. L., B. J. Conrath, L. N. Fletcher, P. J. Gierasch, A. A. Simon-Miller, and L. C. Zuchowski (2009b), Mapping potential vorticity dynamics on Saturn: Zonal mean circulation from Cassini and Voyager data, Planet. Space Sci., 57, 1682-1698.

Sánchez-Lavega, A. (1982), Motions in Saturn's atmosphere: Observations before Voyager encounters, Icarus, 44, 1-16.

Sánchez-Lavega, A. (1994), Saturn's Great White Spots, Chaos, 4, 341-353.

Sánchez-Lavega, A., F. Colas, J. Lecacheux, P. Laques, I. Miyazaki, and D. Parker (1991), The Great White Spot and disturbances in Saturn's equatorial atmosphere during 1990, Nature, 353, 397-401.

Sánchez-Lavega, A., J. Lecacheux, F. Colas, and P. Laques (1993), Temporal behavior of cloud morphologies and motions in Saturn's atmosphere, J. Geophys. Res., 98, 18,857-18,872.

Sánchez-Lavega, A., J. Lecacheux, J. M. Gomez, F. Colas, P. Laques, K. Noll, D. Gilmore, I. Miyazaki, and D. Parker (1996), Large scale storms in Saturn's atmosphere during 1994, Science, 271, 631-634.

Sánchez-Lavega, A., J. F. Rojas, J. R. Acarreta, F. Colas, and P. V. Sada (1997), New observations and studies of Saturn's long-lived north polar spot, Icarus, 128, 322-334.

Sánchez-Lavega, A., J. Lecacheux, F. Colas, J. F. Rojas, and J. M. Gomez (1999), Discrete cloud activity in Saturn's equator in 1995, 1996, and 1997, Planet. Space Sci., 47, 1277-1283.

Sánchez-Lavega, A., J. F. Rojas, and P. V. Sada (2000), Saturn's zonal winds at cloud level, Icarus, 147, 405-420.

Sánchez-Lavega, A., R. Hueso, S. Péres-Hoyos, J. F. Rojas, and R. G. French (2004), Saturn's cloud morphology and zonal winds before the Cassini encounter, Icarus, 170, 519-523.

Sánchez-Lavega, A., et al. (2011), Deep winds beneath Saturn's upper clouds from a seasonal long-lived planetary-scale storm, Nature, 475, $71-74$. 


\section{COWLEY AND PROVAN: MAGNETOSPHERIC OSCILLATIONS AND THE GWS}

Sánchez-Lavega, A., T. del Rio-Gaztellurrutia, M. Delcroix, J. Legarreta, J. M. Gómez-Forrellad, R. Hueso, J. E. García-Melendo, S. Pérez-Hoyos, D. Barrado-Navascués, and J. Lillo (2012), Ground-based observations of the long-term evolution and death of Saturn's 2010 Great White Spot Icarus, 220, 561-576.

Sandel, B. R., and A. L. Broadfoot (1981), Morphology of Saturn's aurora, Nature, 292, 679-682.

Sandel, B. R., et al. (1982), Extreme ultraviolet observations from the Voyager 2 encounter with Saturn, Science, 215, 548-553.

Sayanagi, K. M., and A. P. Showman (2007), Effects of a large convective storm on Saturn's equatorial jet, Icarus, 187, 520-539.

Sayanagi, K. M., U. A. Dyudina, S. P. Ewald, G. Fischer, A. P. Ingersoll, W. S. Kurth, G. D. Muro, C. C. Porco, and R. A. West (2013a) Dynamics of Saturn's great storm of 2010-2011 from Cassini ISS and RPWS, Icarus, 223, 460-478.

Sayanagi, K. M., U. A. Dyudina, S. P. Ewald, G. Fischer, G. D. Muro, and A. P. Ingersoll (2013b), Cassini ISS observations of Saturn's String of Pearls, Icarus, doi:10.1016/j.icarus.2013.10.032, in press.

Smith, C. G. A. (2006), Periodic modulation of gas giant magnetospheres by the neutral upper atmosphere, Ann. Geophys., 24, 2709-2717.

Smith, C. G. A. (2010), A Saturnian cam current system driven by asymmetric thermospheric heating, Mon. Not. R. Astron. Soc., 410, 2315-2329, doi:10.1111/j.1365-2966.2010.17602.x.

Smith, C. G. A., and A. D. Aylward (2008), Coupled rotational dynamics of Saturn's thermosphere and magnetosphere: A thermospheric modelling study, Ann. Geophys., 26, 1007-1027.

Southwood, D. J. (2011), Direct evidence of differences in magnetic rotation rate between Saturn's northern and southern polar regions, J. Geophys. Res., 116, A01201, doi:10.1029/2010JA016070.

Southwood, D. J., and M. G. Kivelson (2007), Saturn magnetospheric dynamics: Elucidation of a camshaft model, J. Geophys. Res., 112 , A12222, doi:10.1029/2007JA012254.

Southwood, D. J., and M. G. Kivelson (2009), The source of Saturn's periodic radio emissions, J. Geophys. Res., 114, A09201, doi:10.1029/ 2008JA013800.
Stallard, T. S., S. Miller, L. M. Trafton, T. R. Geballe, , and R. D. Joseph (2004), Ion winds in Saturn's southern auroral/polar region, Icarus, 167, 204-211.

Stallard, T. S., A. Masters, S. Miller, H. Melin, E. J. Bunce, C. S. Arridge, N. Achilleos, M. K. Dougherty, and S. W. H. Cowley (2012), Saturn's auroral/polar $\mathrm{H}_{3}{ }^{+}$infrared emission: The effect of solar wind compression, J. Geophys. Res., 117, A12302, doi:10.1029/2012JA01018201.

Thomsen, M. F., D. B. Reisenfeld, D. M. Delapp, R. L. Tokar, D. T. Young, F. J. Crary, E. C. Sittler, M. A. McGraw, and J. D. Williams (2010), Survey of ion plasma parameters in Saturn's magnetosphere, J. Geophys. Res., 115, A10220, doi:10.1029/2010JA015267.

Vasavada, A. R., S. M. Hörst, M. R. Kennedy, A. P. Ingersoll, C. C. Porco, A. D. Del Genio, and R. A. West (2006), Cassini imaging of Saturn: Southern hemisphere winds and vortices, J. Geophys. Res., 111 E05004, doi:10.1029/2005JE002563.

Wang, Z., D. A. Gurnett, G. Fischer, S.-Y. Ye, W. S. Kurth, D. G. Mitchell, J. S. Leisner, and C. T. Russell (2010), Cassini observations of narrowband radio emissions in Saturn's magnetosphere, J. Geophys. Res., 115, A06213, doi:10.1029/2009JA014847.

Warwick, J. W., et al. (1981), Planetary radio astronomy observations from Voyager 1 near Saturn, Science, 212, 239-243.

Warwick, J. W., D. S. Evans, J. H. Romig, J. K Alexander, M. D. Desch M. L. Kaiser, M. Aubier, Y. Leblanc, A. Lecacheux, and B. M. Pedersen (1982), Planetary radio astronomy observations from Voyager-2 near Saturn, Science, 215, 582-587.

West, R. A., K. H. Baines, E. Karkoschka, and A. Sánchez-Lavega (2009), Clouds and aerosols in Saturn's atmosphere, in Saturn From CassiniHuygens, edited by M. K. Dougherty, L. W. Esposito, and S. M. Krimigis, pp. 161-179, Springer Publ., Netherlands.

Ye, S.-Y., D. A. Gurnett, J. B. Groene, Z. Wang, and W. S. Kurth (2010), Dual periodicities in the rotational modulation of Saturn narrowband emissions, J. Geophys. Res., 115, A12258, doi:10.1029/2010JA015780.

Zarka, P., L. Lamy, B. Cecconi, R. Prangé, and H. O. Rucker (2007), Modulation of Saturn's radio clock by solar wind speed, Nature, 450, 265-267, doi:10.1038/nature06237. 